\title{
RELIGIONE E IDEOLOGIA NEL GIOVANE AGRIPPA: APPUNTI SULLA VOCAZIONE INTELLETTUALE
}

\author{
RELIGION AND IDEOLOGY IN THE YOUNG AGRIPPA: \\ NOTES ON THE INTELLECTUAL VOCATION
}

\author{
DARIO GURASHI \\ ISTITUTO NAZIONALE Di STUDI SUL RINASCIMENTO, FiRENZE
}

\begin{abstract}
La riforma della cultura promossa da Cornelio Agrippa richiede una riorganizzazione della teologia tradizionale. Recuperando la prisca theologia, Agrippa mira ad emancipare l'esegesi delle Scritture dalla filosofia scolastica, responsabile di aver corrotto il significato autentico della Parola di Dio tramite un impiego indisciplinato della ragione a scapito della fede. Ripensare l'approccio alla Bibbia significa allora ridefinire il concetto di teologia: in quanto meditazione sulla parola divina, essa dovrà abbandonare le dispute dialettiche per tradursi in uno stile di vita contrassegnato dalla virtù e dall'integrità della fede in Cristo. Il programma di Agrippa, dunque, intende dimostrare l'incompetenza dei teologi scolastici e allo stesso tempo rivendica per il pensatore laico la libertà di interpretare le fonti della vera sapienza.
\end{abstract}

Parole chiave

Teologia, magia, ermetismo, esegesi, ragione.

Abstract

The reform of culture promoted by Cornelius Agrippa requires a rearrangement of the traditional theological thinking. By recovering the prisca theologia, Cornelius Agrippa intends to free the exegesis of the Scriptures from Scholastic philosophy, guilty of corrupting the meaning of the Word of God through an unruly use of the human reason at the expense of faith. Rethinking the approach to the Bible also means redefining the concept of theology: since it constitutes an investigation into the divine word, theology must abandon dialectical disputes in order to become a way of life characterized by virtue and integrity of faith in Christ. Agrippa's goal is to show on one hand the ineptitude of the Scholastic theologians on the other to claim for the lay thinker the freedom to interpret the sources of true wisdom.

Key Words

Theology, Magic, Hermeticism, Exegesis, Reason.

Mediterranea. International journal on the transfer of knowledge, 5 (2020), p. 153-192 ISSN: 2445-2378

(C) The author(s). Published by UCOPress. Cordoba University Press. All rights reserved. 
La riflessione teorica che Heinrich Cornelius Agrippa von Nettesheim sviluppa negli anni di soggiorno in Italia costituisce un passaggio cruciale per determinare la missione intellettuale che un teologo è chiamato a incarnare. La teologia, interesse duraturo nella maturazione filosofica di Agrippa, assume in questa fase, tra il 1511 e il 1518, un profilo ideologico e una chiarezza programmatica a cui l'autore resterà sostanzialmente fedele nel corso di tutta la sua vicenda intellettuale. ${ }^{1}$ Infatti il programma di riforma magico-religiosa della cultura, che

1 Gli studi critici sulla figura e sull'opera di Agrippa von Nettesheim (1486-1535) costituiscono il prodotto maturo della storiografia filosofica novecentesca, inaugurata dalle ricerche di studiosi del calibro di Eugenio Garin, Daniel Pickering Walker, Frances Amelia Yates e François Secret tra gli anni ' 50 e ' 60 del secolo scorso. Tali ricerche hanno promosso un ripensamento generale delle categorie storiografiche adoperate nella storia della scienza e nella storia delle idee, sviluppando una linea di indagine che riconosce nella Magia, nell'Ermetismo e nella Qabbalah, spazi di riflessione filosofico-religiosa caratteristici del Rinascimento europeo. Sotto questo profilo gli scritti di Agrippa, che di questi temi presentano un approfondimento dettagliato - in dialogo costante con precursori come Marsilio Ficino, Giovanni Pico della Mirandola, Ludovico Lazzarelli, Johannes Reuchlin, Paolo Ricci, Francesco Zorzi - hanno assunto nel corso del Novecento un valore centrale per chiarire aspetti controversi della filosofia del Rinascimento. Pur nella diversità degli esiti interpretativi, che non è possibile delineare in una nota, questo approccio metodologico è stato sviluppato ininterrottamente nei contributi di riferimento che qui vengono richiamati brevemente. Le ricerche di Charles Garfield Nauert e i numerosi studi di Paola Zambelli hanno evidenziato a più riprese i fattori innovativi del pensiero di Agrippa, rintracciando nelle sue opere la volontà di affrontare un'epoca di crisi della cultura e di decadenza della spiritualità, attraverso una riforma dei saperi che abbia la magia per fondamento; ossia di caldeggiare una religiosità di carattere neoplatonico ed ermetico, fondata sul recupero del patrimonio sapienziale della magia antica, nell'ottica di contrastare i conflitti religiosi innescati da un lato dalla corruzione della teologia contemporanea, dall'altro dalla Riforma di Lutero. cf. CHARLES G. NAUERT, « Magic and Skepticism in Agrippa's thought ", Journal of the History of Ideas, 18/2 (1957), p. 161-182; ID., " Agrippa in Renaissance Italy: the Esoteric Tradition ", Studies in the Renaissance, 6 (1959), p. 195-222; ID., Agrippa and the Crisis of Renaissance Thought, University of Illinois Press, Urbana 1965 (Illinois Studies in the Social Sciences, 55); Paola Zambelli, «A proposito del De vanitate scientiarum et artium di Cornelio Agrippa », Rivista Critica di Storia della Filosofia, 15/2 (1960), p. 166-180; EAD., "Cornelio Agrippa nelle fonti e negli studi recenti », Rinascimento, 8 (1968), p. 169-199; EAD., "Cornelio Agrippa, Erasmo e la teologia umanistica ", Rinascimento, 10 (1970), p. 29-88; EAD., " Magic and Radical Reformation in Agrippa of Nettesheim ", Journal of the Warburg and Courtauld Institutes, 39 (1976), p. 69-103. Successivamente i notevoli studi condotti da Vittoria Perrone Compagni, a cui si aggiungono le pregiate edizioni di testi agrippiani, che sviluppano un'iniziativa editoriale avviata da Paola Zambelli, hanno approfondito gli esiti della riforma magico-religiosa della cultura che Agrippa propugna, analizzando le matrici autenticamente filosofiche del suo programma. Le ricerche di Perrone Compagni - che hanno trovato in seguito nei saggi di Marc van der Poel e di Christopher I. Lehrich interessanti ripercussioni - hanno dimostrato in primo luogo quanto fosse parziale e inadeguato il paradigma interpretativo che classificava il pensiero di Agrippa nell'ambito di una scelta tra 'scetticismo' e 'occultismo, secondo una tendenza storiografica ampiamente attestata nella critica novecentesca (G. Rossi, E. Cassirer, R. Stadelmann, 
Agrippa intraprende già nel corso della prima stesura del De occulta philosophia (1510), prevede non solo l'approfondimento di una meditazione sull'uomo ispirata alla letteratura ermetica e neoplatonica, bensì soprattutto il riassetto delle coordinate teologiche tradizionali. La critica ha ampiamente sottolineato, per quanto riguarda questa fase del pensiero di Agrippa, l'influenza considerevole del programma ficiniano di sottoporre a indagine la cosiddetta prisca theologia, muovendo dallo studio e dalla trasmissione delle fonti più antiche. ${ }^{2}$ Recuperare le verità divine tramandate nelle opere dei vetustissimi scriptores riveste, a ben vedere, un significato del tutto peculiare nel progetto intellettuale di Agrippa, traducendosi nella volontà di rintracciare nella sua purezza la prisca sapientia, ossia quel sapere incorruttibile che, dignificato dalla sua origine sovrumana e dal

L. Thorndike, H. Haydn, C. G. Nauert, G. H. Daniels); in secondo luogo quanto fosse essenziale ridefinire le categorie storiografiche stesse, al fine di focalizzare, nell'apparente contraddittorietà dei pronunciamenti, l'unità del percorso di Agrippa, l'omogeneità del suo messaggio filosofico, rintracciabile, ad esempio, nel concetto di 'rigenerazione ermetica', come ha sostenuto anche Michael H. Keefer; cf. PaOla Zambelli, "Cornelio Agrippa. Testi scelti », in Eugenio Garin, Mirella Brini, Cesare Vasoli, Paola Zambelli (eds.), Testi umanistici su l'ermetismo. Testi di Ludovico Lazzarelli, F. Giorgio Veneto, Cornelio Agrippa di Nettesheim, Fratelli Bocca, Roma 1955 [Archivio di filosofia: organo della Società filosofica italiana, 1/1 (1955)], p. 105-162; EAD., « Agrippa di Nettesheim. Dialogus de homine, prima ed. », Rivista critica di storia della filosofia, 13, 1, 1958, p. 47-71; EAD., «Cornelio Agrippa. Scritti inediti e dispersi », Rinascimento, 5 (1965), p. 195323; Vittoria Perrone Compagni, "Una fonte di Cornelio Agrippa: il De harmonia mundi di Francesco Zorzi », Annali dell'Istituto di filosofia, 4 (1982), p. 45-74; EAD., «Il De occulta philosophia di Cornelio Agrippa », in Guido CANZIANI, GIANNI PAgANINI (eds.), Le edizioni dei testi filosofici e scientifici del '500 e del '600. Problemi di metodo e prospettive di ricerca. Gargnano sul Garda, 13 aprile 1985, Franco Angeli, Milano 1986 (Filosofia e scienza nel Cinquecento e nel Seicento. Serie 1, Studi, 29), p. 99111; EAD., "Introduction 》, in CoRnelius AgripPA AB NETTEShEIM, De occulta philosophia libri tres, Leiden - New York - Köln, Brill 1992 (Studies in the History of Christian Thought, 48), p. 1-50; EAD., "Riforma della magia e riforma della cultura in Agrippa », I Castelli di Yale, 2/2 (1997), p. 115-140; EAD., " Dispersa intentio: Alchemy, Magic and Scepticism in Agrippa », Early Science and Medicine, 5/2 (2000), p. 160-177; EAD., "Astrologia e filosofia occulta in Agrippa », Rinascimento, 41 (2001), p. 93-111; EAD., " Abracadabra: le parole della magia (Ficino, Pico, Agrippa) », Rivista di estetica, 42 (2002), p. 105-130; EAD., Ermetismo e Cristianesimo in Agrippa. Il 'De triplici ratione cognoscendi Deum', Polistampa, Firenze 2005 (Hermetica Mediaevalia, 3); EAD., «Il De occulta philosophia di Cornelio Agrippa. Parole chiave: uomomicrocosmo, prisca theologia, cabala, magia », Bruniana \& Campanelliana, 13/2 (2007), p. 429-448; EAD., "Tutius ignorare quam scire. Cornelius Agrippa and Scepticism ", in Gianni Paganini, José R. Maia Neto (eds.), Renaissance Scepticisms, Springer, Dordrecht 2009 (Archives internationales d'histoire des idées, 199), p. 91110; MARC VAN DER PoEL, "Agrippa von Nettesheim and Rhetoric: An Examination of the Declamatio de originali peccato», Humanistica Lovaniensia, 39 (1990), p. 177-206; ID., Cornelius Agrippa. The Humanist Theologian and His Declamation, Brill, Leiden 1997 (Brill's Studies in Intellectual History, 77); CHRISTOPHER. I. LeHRICH, The Language of Demons and Angels. Cornelius Agrippa's Occult Philosophy, Leiden, Brill 2003 (Brill's Studies in Intellectual History, 119); MicHAEL. H. KEEFER, « Agrippa's Dilemma: Hermetic 'Rebirth' and the Ambivalences of De vanitate and De occulta philosophia », Renaissance Quarterly, 41 (1988), p. 614-653.

2 Cf. in particolare Nauert, Agrippa and the Crisis, p. 43-46; Perrone Compagni, Ermetismo e Cristianesimo, p. 12-36; EAD., «Il De occulta philosophia », p. 435-439. 
suo valore etico-religioso, è stato tramandato nel corso dei secoli dalle voci più autorevoli della teologia. Questo bagaglio di conoscenza è capace, qualora restaurato nella dignità della sua dottrina, di legittimare una riforma della cultura contemporanea. ${ }^{3}$ L'approccio di Agrippa assume, dunque, la forma di un'esplorazione sapienziale, concepita per attingere alle fonti pagane, cronologicamente più remote e ideologicamente consone all'insegnamento cristiano, una dottrina primigenia, espressione di una medesima verità, sempiterna e soprattutto salvifica, necessaria per ricomporre e rinnovare la teologia cristiana riscattandone il messaggio originario. ${ }^{4}$

3 Sul significato marcatamente religioso della filosofia, che fonda in Agrippa il ricorso al modello sapienziale della pia philosophia, cf. PerRone Compagni, « Il De occulta philosophia », p. 435-436: « La radice 'teologica' del sapere umano è per Agrippa nient'altro che il riconoscimento del rapporto che la filosofia deve intrattenere con Dio. [...] Il modello di questo atteggiamento del filosofare, che unisce sapientia e pietas, è la tradizione di antichi teologi che seppero leggere il libro della natura, scritto anch'esso digito Dei, con gli occhi fissi, più che al contenuto, all'autore e riconobbero nella realtà fisica il cosmico processo d'amore che ha il suo centro nel Bene eterno. Anche la ragione pagana era perfettamente alfabetizzata per interpretare l'insieme dei segni comunicativi con cui Dio si faceva conoscere nelle cose del mondo. La grandezza dei prisci theologi consiste nell'aver condotto la lettura non affidandosi ai sensi, ma all'attività contemplativa della mens, giungendo così a intuire quelle verità a cui la ragione cristiana, arricchita e sostenuta dalla Rivelazione, ha ora pieno accesso; ma anche nell'aver saputo accompagnare il loro atto conoscitivo al culto devoto del Principio».

4 In un saggio recente Thomas Leinkauf ha analizzato i modelli di approccio allo studio, al recupero e alla trasmissione delle dottrine dei filosofi antichi, specialmente i Presocratici, durante il Rinascimento in Europa, distinguendo due metodi esemplari di comprensione della filosofia antica che hanno assunto, per i pensatori rinascimentali, il valore di veri e propri paradigmi storiografici. Nel primo caso si tratta del modello che si appella alla prisca scientia, impiegato soprattutto dagli Scolastici, in cui l'assenza di una preoccupazione storico-filologica determina una considerazione strettamente teoretica della questione: le dottrine degli antichi figurano come serbatoio di nozioni, precetti, osservazioni, alle quali attingere per risolvere essenzialmente questioni concettuali. Nel secondo caso si individua il modello della prisca sapientia, propugnato principalmente dagli umanisti, che ascrive ai pensatori antichi la trasmissione di una sapienza originaria e divina, di carattere religioso, promotrice, in quanto conoscenza salvifica, di un rinnovamento dell'esistenza umana. Sotto questo profilo, le scelte di Agrippa sono pienamente inquadrabili nell'ambito di quest'ultimo approccio; cf. THомAs LEINKAUf, « Prisca scientia versus prisca sapientia. Zwei Modelle des Umgangs mit der Tradition am Beispiel des Rückgriffs auf die Vorsokratik im Kontext der frühneuzeitlichen Debatte und der Ausbildung des Kontinuitätsmodell der prisca sapientia bzw. philosophia perennis », Mediterranea. International Journal on the Transfer of Knowledge, 2 (2017), p. 136: «Der Gedanke der prisca sapientia beinhaltet nicht nur den - einem Vorurteil oft nicht unähnlichen - Gedanken von der apriorischen, unabweisbaren, an Offenbarung oder intuitive Evidenz geknüpften Dignität des alten, ja ältesten Denkens gegenüber jeglichem sich nicht aus diesem selbst herleitenden späteren, bis hin zur Zeitgenossenschaft gehenden Denkens, sondern er ist unauflöslich verbunden mit dem zweiten Gedanken, daß die prisca sapientia nicht schlechthin vergangen und dadurch auch ebenso unausweichlich nur ex post und anachronistisch für jeden Späteren zugänglich sei, sondern daß sie, umgekehrt, kraft ihrer Dignität und Autorität, sich durch einen kontinuierlichen Überlieferungsstrom gegenwärtig gehalten habe. Dadurch könne ihr 
L'operazione, eminentemente umanistica, è radicata nella convinzione di poter associare, ad esempio, il più autorevole degli antichi sapienti, Ermete Trismegisto, ed il più importante dei teologi cristiani, San Paolo, in un'esperienza spirituale condivisa. Quasi tutti gli opuscoli che Agrippa compone durante il soggiorno italiano - se si esclude la prolusione sul simposio di Platone, risalente probabilmente al 1512, ${ }^{5}$ bisogna ricordare gli scritti redatti tra il 1515 e il 1516: l'Oratio habita Papiae in praelectione Hermetis Trismegisti De potestate et Sapientia Dei, il Dialogus de homine e il De triplici ratione cognoscendi Dei - rimarcano per l'appunto la volontà di collocare, tramite l'accertamento della consonanza teorica tra San Paolo ed Ermete Trismegisto, l'eredità sapienziale del Corpus Hermeticum all'interno della tradizione dottrinale cristiana. ${ }^{6}$ La conformità delle dottrine ermetiche con il messaggio evangelico è giustificata, sul piano ideologico, riconoscendo al Trismegisto un'indiscussa autorità teologica per il fatto di aver attinto alla medesima fonte di verità, la rivelazione di Cristo, che ispira e permea l'epistolario paolino. ${ }^{7}$ Come attesta soprattutto il dittico dei già menzionati

spezifischer Gehalt [...] sozusagen direkt funktionalisiert werden für Bedürfnisse der eigenen Gegenwart: die prisca sapientia ist zugleich auch der Gehalt dessen, was eine sapientia renovata oder restituta als Antidot gegen nicht gewollte Usurpationen des religiösen Bewußtseins der eigenen Zeit fungibel macht ».

5 AGRIPPA, Oratio in Praelectionem Convivij Platonis, in ID., Orationes X, fol. A2r-B3r.

6 Sull'interesse giovanile di Agrippa per la letteratura ermetica, cf. WOUTER J. HANEGRAAFF, « Better than Magic. Cornelius Agrippa and Lazzarellian Hermetism », Magic, Ritual and Witchcraft, 4/1 (2009), p. 6: «Agrippa's 'hermetic' period runs from 1509 to 1518, the last seven years of which he spent in Italy. It begins whith his lectures on Reuchlin at the University of Dôle in 1509, which unfortunately have not been preserved. The first documented evidence we have of his hermetic interests is the first draft of De occulta philosophia from 1510. Five years later Agrippa lectured on Ficino's Pimander in Pavia. Again, these lectures themselves are lost, but we still have the introduction, known as the Oratio habita Papiae in praelectione Hermetis Trismegisti De potestate et Sapientia Dei. It was followed one year later, in 1516, by a Dialogus de Homine of which we have only the first part, and, finally, by a complete text known as De triplici ratione cognoscendi Deum ».

Nella prolusione accademica dedicata al Corpus Hermeticum, che Agrippa declama a Pavia nel 1515, viene affermata l'identificazione del Pimander ermetico con Cristo stesso; cf. ZAMBELLI, Testi scelti, p. 125: « polliceorque vobis effecturum me totis viribus, ut nihil sit in suscepta materia tam difficile, tam intricatum, tam obscurum, tamque arduum cuicunque facultati aut scientiae ea traditio consonet, quod me interprete non assequamini omnes, intelligentia, favente nobis ipso ter maximi Mercurij Pimandro, mente divinae potentiae domino videlicet nostro Jesu Christo Nazareno crucifixo, qui verus Pimander, qui magni consilii Angelus vero mentis lumine illustrat, quem verum Deum et verum hominem, regenerationis autorem confitemur, futurique patrem seculi judicem expectamus ». La fonte dell'identificazione e del passo testuale di Agrippa è Lazzarelli: cf. LODOvicus LAZARELUS, Crater Hermetis, in Contenta in hoc volumine. Pimander Mercurii Trismegisti liber de sapientia et potestate Dei. Asclepius Eiusdem Mercurii Liber de Voluntate Diuina. Item Crater Hermetis, a Lazarelo Septempedano, Paris 1505, fol. 60v: " qui magni consilii angelus vero mentes lumine illustravit, qui princeps pacis hominem deo conciliavit; quem verum deum et verum hominem profitemur ac futuri patrem seculi iudicemque expectamus. Ipse qui in Hermetis mente Pimander erat, in me Christus Ihesus incolatum facere dignatus est consolator 
Dialogus de homine e De triplici ratione cognoscendi Deum, ${ }^{8}$ l'approccio di Agrippa al dibattito religioso riprende e innova in chiave ermetico-paolina una lezione metodologica promossa dai pensatori 'ermetici' del tardo Quattrocento - basti menzionare, oltre a Marsilio Ficino, Giovanni Pico della Mirandola e Lodovico Lazzarelli, tra i più autorevoli, ${ }^{9}$ ossia gli autori che maggiormente occupano gli studi di Agrippa nel periodo di formazione - con l'intento di coniugare, attraverso una strategia di calibrato equilibrio teorico, Ermetismo e Cristianesimo, riconosciuti entrambi depositari, al pari degli scritti di Platone e dei suoi seguaci tardo-antichi, di un insegnamento filosofico e soteriologico omogeneo. ${ }^{10}$

Se si considera un profilo teorico più ampio, il recupero della prisca theologia permette ad Agrippa di inserirsi pienamente nel vasto dibattito che coinvolge, durante le prime decadi del Cinquecento, umanisti tedeschi come Sebastian Brant, Jakob Wimpfeling, Johann Reuchlin, Ulrich von Hutten, i quali si

eternus »; cf. PerRone Compagni, «Ermetismo e Cristianesimo », p. 32-33; HANEgraAfF, « Better than magic », p. 10, 16-17; ID., " Heinrich Cornelius Agrippa », in Christopher PARTRIDGE (ed.), The Occult World, Routledge, London-New York 2016, p. 93-94.

8 Per il De homine cf. Paola Zambelli, «Agrippa di Nettesheim. Dialogus de homine ", (prima ed.), Rivista critica di storia della filosofia, 13 (1958), p. 47-71; una seconda edizione, riveduta e aggiornata, è stata pubblicata in EAD., "Cornelio Agrippa. Scritti inediti e dispersi ", Rinascimento, 5 (1965), p. 294-304. I richiami al Dialogus sono tratti dall'edizione aggiornata. Per il De triplici ratione, cf. PERRONE COMPAGNI, Ermetismo e Cristianesimo, p. 52-84.

9 RaphaEl EbGi (ed.), Umanisti italiani. Pensiero e Destino, Einaudi, Torino 2016 (I millenni), p. 341350.

10 Perrone Compagni, « Il De occulta philosophia », p. 436; cf. EAD., Ermetismo e Cristianesimo, p. 52-53: «Ma lo scritto del 1516 [scil. De triplici ratione cognoscendi deum] rappresenta anche il primo luogo di esplicito ricongiungimento delle due autorità fondamentali del 'cristianesimo' di Agrippa: il profeta Ermete e l'apostolo Paolo - le figure che insieme, seppure in modi diversi, mostrano l'unico volto della religiosità umana e la continuità della sapienza classico-cristiana di contro alle spinte disgregatici di una ragione che procede senza avvertire né i propri limiti, né la necessità di un superiore fondamento di certificazione, né l'esigenza di tradursi in un sapere attivo, ossia socialmente benefico ». Questa convergenza ermetico-paolina è verificabile testualmente in alcuni passi del De triplici ratione e del Dialogus de homine. A titolo esemplificativo, cf. AgRIPPA, De triplici ratione cognoscendi Deum, in PERRONE COMPAGNI, Ermetismo e Cristianesimo, p. 96-98: «Unde praecipitantur in turpes sensuum illecebras, in letiferas peccatorum sordes, corruunt in omne flagitium et in naturae perversionem subiiciunturque 'cupiditatum imperio, ad quarum expletionem', ut inquit Hermes, 'ardenti quodam impetu perferuntur rituque ferarum immoderato et irascuntur et cupiunt; quodque deterius est, nec finem imponunt libidini ullum, nec malorum inveniunt passionumque terminum'; quae omnia Paulus in Epistola ad Romanos clare edocet »; ID., Dialogus de homine, p. 303: « Oportet autem te (ut inquit Hermes) vestem quam circumfers exuere, indumentum inscitie, pravitatis fundamentum, corruptionis vinculum, vivam mortem, sensitivum cadaver, sepulcrum circumfertile, domesticum inimicum, de quo ait Christus: qui post me vult venire, abneget semetipsum ac tollat crucem suam et sequatur me; que crux nil aliud est quam corpus hoc materiale, quod in similitudinem crucis geritur. Hoc nos abnegare et relinquere oportet, ut cum Christo ad pristinam <in>mortalitatem revertamur. Hinc Paulus ait: cupio dissolvi et esse cum Christo ». 
interrogano a più riprese sulla crisi spirituale che investe l'Europa nel suo complesso tramite un'analisi serrata delle cause per cui dilaga la corruzione dei costumi religiosi. In particolare, negli anni che precedono la diffusione del movimento luterano, la produzione di Agrippa è contrassegnata dal progetto di ricostituire il sapere magico su nuovi fondamenti. L'impegno in questa direzione, incoraggiato anche dal confronto con Johannes Trithemius, risulta profondamente influenzato non solo dalla volontà di rivalorizzazione una disciplina, la magia, screditata lungo i secoli e ripetutamente gravata dall'onta dell'eresia, ${ }^{11}$ bensì soprattutto dall'urgenza di rifondare l'esperienza religiosa, ricercando, nei termini di una vera e propria restauratio christiana, soluzioni filosofiche che, custodite nel patrimonio che la sapienza occulta dischiude, risultino efficaci ad arrestare e contrastare la crisi spirituale contemporanea. ${ }^{12}$

A tal proposito, ripercorrendo una caratterizzazione ormai classica delle prerogative del mago rinascimentale - illustrata da Marsilio Ficino nell'Apologia al De vita coelitus comparanda - Agrippa avrebbe sottolineato, nell'avvertenza al lettore che apre la redazione finale dei tre libri De occulta philosophia (1533), l'orientamento cristiano e il fondamento evangelico di una professione ampiamente contestata:

Per i dotti mago non significa stregone, superstizioso, demoniaco, bensì sapiente, sacerdote, profeta; le Sibille fuono maghe, e perciò profetizzarono con la massima evidenza l'avvento di Cristo. Certo anche i Re Magi, comprendendo i meravigliosi arcani del mondo, seppero che era nato Cristo, il creatore del mondo, e primi fra tutti vennero ad adorarlo. Il nome stesso di magia è accettato dai filosofi, lodato dai teologi e gradito persino al Vangelo. ${ }^{13}$

11 Sulla vindicatio magiae cf. la lettera dedicatoria a Trithemius in AGRIPPA, Ioanni Tritemio Henricus Cornelius Agrippa, in ID., De occulta philosophia, p. 70: "Mirabar admodum neque minus etiam indignabar neminem hactenus extitisse, qui tam sublimem sacramque disciplinam ab impietatis crimine vindicasset aut illam nobis pure syncereque tradidisset siquidem quos ex recentioribus ego vidi [...] cum se magiam tradere pollicentur, nonnisi aut deliramenta quaedam, nulla ratione subnixa, aut superstitiones probis omnibus indignas praestiterunt. Hinc concitus est in me spiritus meus atque propter ipsam cum admirationem tum indignationem volui et ego philosophari, non illaudabile opus me facturum existimans [...] si magiam ipsam vetustam sapientumque omnium disciplinam ab impietatis erroribus redimitam purgatamque et suis rationibus adornatam restituerem et ab iniuria calumniantium vindicarem ».

12 PeRrone COMPAGNI, «Riforma della magia », p. 120: "La restaurazione della magia si traduce, in ultima analisi, nel recupero della sua matrice originariamente religiosa; l'illuminazione, che si realizza per libera iniziativa divina in una mente preparata a riceverla, determina la metamorfosi del filosofo in mago e la trasformazione del sapere razionale in potere taumaturgico ».

13 AgripPA, Ad lectorem, in ID., De occulta philosophia libri tres, ed. PERRONE COMPAGNI, p. 65: «magum apud literatos viros non maleficum, non superstitiosum, non daemoniacum sonare, sed sapientem, sed sacerdotem, sed prophetam; Sybillas magas fuisse, proinde de Christo tam apertissime prophetasse; iam vero et Magos ex mirabilibus mundi arcanis ipsius mundi autorem 
La determinazione del valore della magia, in cui sia ascritta al mago una funzione sacrale, oltreché sapienziale, per il contesto teologico cristiano, non rappresenta l'esito di una riflessione occasionale, bensì una premessa $o$, piuttosto, una preoccupazione teorica tenacemente viva nel pensiero di Agrippa; essa risale in realtà al periodo della primissima formazione, tra Colonia, Parigi e Londra (14991510), e prosegue concretizzandosi durante tutto il soggiorno in Italia. ${ }^{14}$ Accolta, infatti, la lezione di Erasmo da Rotterdam, di cui si dichiarerà strenuo difensore e appassionato seguace, anche il filosofo di Nettesheim persegue un ideale di rinnovamento del culto religioso tramite il recupero della devozione originaria e autenticamente cristiana - di cui offrono vivida testimonianza i primi discepoli di Gesù e i Padri della Chiesa - come presupposto per rifondare la teologia nel suo insieme e, a partire dalla teologia, riorganizzare tutto il sapere. ${ }^{15}$

Christum cognovisse natum omniumque primos venisse ad illum adorandum ipsumque magiae nomen acceptum philosophis, laudatum a theologis, etiam ipsi Evangelio non ingratum »; cf. Marsilius Ficinus, Apologia, in ID., Opera omnia, Henricuspetrus, Basileae 1576, t. I, p. 573: « Quid igitur expavescis Magi nomen formidolose? Nomen evangelio gratiosum, quod non maleficum et veneficum, sed sapientem sonat et sacerdotem. Quidnam profitetur Magus ille, venerator Christi primus? Si cupis audire, quasi quidam agricola est, certe quidam mundicola est. Nec propterea mundum hic adorat, quemadmodum nec agricola terram, sed sicut agricola humani victus gratia ad aerem temperat agrum, sic ille sapiens, ille sacerdos gratia salutis humanae inferiora mundi ad superiora contemperat; atque sicut ova gallinae, sic opportune terrena subicit fovenda coelo. Quod efficit semper ipse Deus, et faciendo docet suadetque facere, ut a superis infima generentur et moveantur atque regantur $»$.

14 La biografia di Agrippa è stata oggetto di tre studi importanti, dei quali il più recente costituisce ancora oggi la ricostruzione più attendibile e aggiornata: cf. HENRY MORLEY, Cornelius Agrippa. The life of Henry Cornelius Agrippa von Nettesheim, doctor and knight, commonly known as a magician, 2 vols., Chapman and Hall, London 1856; AugusTE PROST, Les sciences et les arts occultes au xvi e siècle: Corneille Agrippa. Sa vie et ses oeuvres, 2 vols., Champion, Paris 1882; NAUERT, Agrippa and the Crisis. 1965. Per quanto riguarda il periodo di formazione che precede il trasferimento in Italia (14991510) è utile ripercorrere brevemente alcune informazioni biografiche. Agrippa si forma nella prima decade del Cinquecento presso l'università di Colonia sotto la guida di Andreas e Jacobus Canter, rinomati docenti di lingua latina, fortemente critici del lessico scolastico e appassionati cultori dell'arte lulliana. Successivamente conclude gli studi legali in utroque iure e ottiene il dottorato in medicina. Ancora a Colonia entra a far parte del circolo di Hermann von Neuenahr e Johannes Caesarius, intellettuali pubblicamente impegnati nella difesa di Johannes Reuchlin contro i Domenicani durante la disputa sulla conservazione dei libri ebraici. Nel 1509, a ventitré anni, tiene alcune lezioni sul De verbo mirifico di Reuchlin a Dôle in Borgogna. Qualche tempo dopo, nel 1510, per il tramite degli umanisti coloniensi, Agrippa incontra a Würzburg l'abate Trithemius, grazie alle cui sollecitazioni prenderà corpo l'idea di restaurare la magia. A Trithemius l'autore dedicherà la prima stesura del De occulta philosophia. Nello stesso anno Agrippa si reca a Londra per seguire le lezioni del teologo John Colet, sotto la cui supervisione studia approfonditamente le Epistole di San Paolo. Cf. in particolare ZAmBELLI, Cornelio Agrippa nelle fonti, p. 174-177; VAN DER POEL, Cornelius Agrippa, p. 15-21.

15 Sull'influenza degli scritti di Erasmo nell'evoluzione del pensiero di Agrippa, cf. soprattutto ZAMBElli, « Cornelio Agrippa, Erasmo », p. 29-88; EAD., « Scritti inediti e dispersi », p. 198-199. 
Tuttavia, a differenza di Erasmo, la strategia di Agrippa prevede che il traguardo di rinascita spirituale sia raggiunto non solo riscoprendo la pietas neotestamentaria, nonché riesaminando il contributo dottrinale della Patristica, bensì soprattutto integrando nel paradigma teologico cristiano la pia philosophia degli antichissimi sapienti, specialmente la religiosità e il tragitto sapienziale descritti in dettaglio nel Corpus Hermeticum. ${ }^{16}$ L'orientamento della ricerca in questa direzione è ispirato anche all'opera di esegesi e diffusione degli Hermetica promossa in particolar modo da Jacques Lefèvre d'Étaples, la cui edizione parigina del 1505 - che includeva il Pimander tradotto da Ficino, l'Asclepius attribuito ad Apuleio e il Crater Hermetis composto da Lazzarelli - sarà presente costantemente sullo scritto di Agrippa, assieme ad altre edizioni dello Stapulensis, quali, ad esempio, le Epistole di Paolo, gli scritti di Lullo e di Cusano. ${ }^{17}$ Coerentemente con il suo progetto di studio, commento e trasmissione della più antica sapienza pagana, Agrippa avrebbe dedicato persino un ciclo di lezioni universitarie ai trattati ermetici. In veste di professore di teologia, assunto a Pavia nel 1515, avrebbe esposto e commentato il Pimander, giudicando i trattati del Trismegisto un insegnamento propedeutico alla comprensione dei più reconditi misteri della religione:

Parliamo adesso della sua opera, intitolata Pimander, o della sapienza e potenza di Dio. È un libro ricchissimo di eleganza stilistica, ricolmo di massime, pieno di grazia e di decoro, pieno di sapienza e di misteri. Ci insegna a conoscere Dio, il mondo, la mente, i due demoni, l'anima, l'ordine della provvidenza, la necessità del fato e da dove essa provenga, la legge di natura, il diritto degli uomini, la religione, le istituzioni sacre, i riti, i templi, le tradizioni e i misteri sacri. Ci istruisce inoltre sulla conoscenza di se stessi, sull'ascesa dell'intelletto, sulle preghiere arcane, sul connubio divino, sul sacramento della rigenerazione e, per riassumere brevemente il tutto, ci insegna a conoscere e comprendere le leggi delle cerimonie consacrate, $\mathrm{i}$ precetti dei riti sacri, le norme delle pratiche religiose, come dobbiamo ottenere la

16 Perrone Compagni, «Il De occulta philosophia », p. 436: «Sono i testi ermetici a sostanziare il costante appello di Agrippa a una non più procrastinabile reformatio (della cultura, della magia, della morale contemporanea) che si costituisca come premessa per l'affermarsi di una 'filosofia incontaminata', come appunto la definiva l'Asclepius - una filosofia, cioè, che sappia riconoscere in Dio il suo centro di irraggiamento e il suo punto di ritorno »; cf. VAN DER POEL, Cornelius Agrippa, p. 264: «On the whole, he [scil. Agrippa] shows himself to be a genuine humanist, who detests the moral and intellectual climate then prevailing in the Church, although his emphasis on spiritual introspection and the important role of the prisca theologia in his theology secure for him a special place among the humanists ».

17 Sulla diffusione del Corpus Hermeticum in Francia e sull'opera editoriale di Lefèvre d'Étaples, cf. Daniel P. Walker, The Ancient theology. Studies in Christian Platonism from the Fifteenth to the Eighteenth Century, Duckworth, London 1972, p. 63-131. 
felicità e come dobbiamo coltivare correttamente la nostra mente, con la quale soltanto possiamo apprendere la verità. ${ }^{18}$

La decisione di presentare le dottrine di Ermete come un modello per ripensare il valore della teologia cristiana, nonché il ruolo sociale di chi la esercita, non ha, dunque, un fondamento erudito; non è semplicemente frutto dell'entusiasmo umanistico per la letteratura antica, ossia per un sapere alternativo alla cultura ufficiale. La riscoperta rinascimentale dell'ermetismo, e con esso la riorganizzazione della magia, che in Agrippa trova uno degli esponenti più radicali, ha in realtà un significato di rinnovamento marcatamente religioso e politico. L'antropologia elaborata nelle pagine dell'Asclepius latino presentava un elogio esemplare della natura umana e della sua collocazione nel cosmo, in cui veniva accertato il ruolo essenziale, metafisico ed epistemologico al contempo, che l'uomo riveste collaborando con Dio nella cura dell'universo e nell'amministrazione del creato. Inoltre, secondo una lezione comune trasmessa nei trattati greci che formano il Pimander, l'autore ermetico celebrava nelle facoltà dell'uomo l'unione armoniosa di religione (fides) e sapienza (mens), concordanza indispensabile per apprendere le verità custodite nella mente di Dio. ${ }^{19}$ Sotto questa luce la rivelazione ermetica proponeva agli occhi di Agrippa un modello etico-filosofico, incarnato per l'appunto dal leggendario Ermete Trismegisto, in cui la vocazione religiosa del sapiente racchiude in una destinazione comune teologia e magia, regalità e giustizia. ${ }^{20}$ Nella prolusione del

18 Il passo è tratto dall'unica testimonianza diretta del corso universitario, il testo edito della prolusione: cf. Agrippa, Oratio habita Papiae, p. 124: «De opere illius [scil. Trismegistus] modo dicamus. Ejus titulus est Pimander, sive de Sapientia et potestate Dei. Est autem liber iste elegantia sermonis refertissumus, copia sententiarum gravissimus, plenus gratiae et decoris, plenus sapientiae et mysteriorum. Docet enim nos, quis Deus, quis mundus, quid mens, quid uterque daemon, quid anima, quis providentiae ordo, quae et unde fati necessitas, quae naturae lex, quod hominum phas, quae religio, quae sacra instituta, ritus, phana, observationes sacraque mysteria. Instruit nos praeterea de cognitione sui ipsius, de ascensu intellectus, de arcanis precibus, de divino connubio, deque regenerationis sacramento, atque, ut paucis cuncta complectar, docet nos rite scire atque callere leges divinorum, phas sacrorum, jusque religionum, et quo pacto felicitatem religione divina debeamus adipisci, quoque pacto mentem nostram, qua sola veritatem apprehendere possumus, rite debeamus excolere ».

19 Ascl. III, fol. 40v (NF 2, pp. 301-301): « Propter hoc, o Asclepi, magnum miraculum est homo, animal adorandum et honorandum. Hoc enim in naturam dei transit, quasi ipse sit deus; hoc demonum genus novit, utpote qui cum eiisdem ortum se esse cognoscat; hoc humane nature partem in se ispso despicit, alterius partis divinitate confisus »; Pim., XII, fol. 30v [NF 1, pp. 178179]: «Illud preterea considera, o fili, quod duo hec soli ex omnibus animalibus homini Deus ipse largitus est, sermonem scilicet atque mentem, quequidem eiusdem (ac immortalitas) premii esse censentur. Iis quisque ad id quod decet utitur, nihil ab immortalibus discrepat. Quinetiam corporis solutus compedibus, ab utrisque ducetur in chorum beatorum simul atque deorum ».

20 PeRrone Compagni, «Dispersa intentio », p. 166: «The magician is the hermetic "perfect philosopher', not because enlightenment endows him with a wisdom that is alternative to 
1515 Agrippa avrebbe fornito una ricostruzione encomiastica della biografia di Ermete Trismegisto (tre volte grandissimo perché al contempo philosophus, sacerdos e rex), traendo spunto dalle genealogie presentate soprattutto nelle fonti coeve come l'Argumentum in librum Mercurii Trismegisti di Ficino, il Crater di Lazzarelli, l'Epistola de astronomiae auctoribus di Agostino Ricci e, probabilmente, il Commentarium redatto da Symphorien Champier alle Diffinitiones Asclepii che Lazzarelli aveva tradotto dal greco: ${ }^{21}$

Dopo aver indagato a fondo tutto il sentiero della sapienza, egli per primo, con poche parole, ma smisuratamente ricche di significato, scrisse a proposito della vera sapienza. Egli, prima di tutti, trattò con somma sapienza della maestà di Dio, dell'ordine degli spiriti, della natura delle anime. Perciò fu definito il primo inventore e fondatore della teologia. Si tramanda che, avendo compreso i fondamenti della religione, scrisse ventiseimilacinquecentoventicinque libri, nei quali rivela arcani mirabili, misteri segretissimi e oracoli meravigliosi. E non parlò soltanto come filosofo, bensì anche come profeta, presagendo molti eventi. Infatti previde e vaticinò la rovina dell'antica religione, la nascita della nuova fede, l'avvento di Cristo, il giudizio futuro, la resurrezione dei morti, il rinnovamento dell'epoca, la gloria dei beati, la punizione dei peccatori. [...] Questo nostro Mercurio, così come superò tutti i filosofi per l'acume della sua intelligenza, allo stesso modo, divenuto sacerdote, superò tutti i sacerdoti per il modo in cui conduceva una vita santa e amministrava il culto del divino. Perciò il rispetto di cui godeva tra gli uomini crebbe a tal punto che gli Egizi lo chiamarono re, dopo Osiride. [...] Ottenuta quindi la dignità regia, Mercurio oscurò la gloria degli altri re per il modo in cui istituiva le leggi, amministrava il regno e compiva grandi imprese. A buon diritto fu definito Trismegisto, ossia tre volte massimo, poiché egli si dimostrò il massimo e sapientissimo filosofo, il massimo e religiosissimo

rational knowledge, but because the sense of human knowledge is disclosed to him by the experience of spiritual regeneration, which also enables him to put science into practice. Yet reason still has the task of assembling the elements of a vast and refined culture, upon which fides bestows dynamism, thereby raising it to the level of knowledge and power. Magic is the result of man's attitude towards God. It is the fruit of a correct orientation and the reward for a willed and trusting adherence to the divine message imprinted in the heart of man. This is why the reform of magic calls primarily for man's spiritual reform, the reorientation of his reason, and a re-appropriated awareness of his destiny ".

21 Cf. Ficinus, Argumentum Marsilij Ficini Florentini in librum Mercurij Trismegisti ad Cosmum Medicem, in Contenta in hoc volumine, fol. 2r-3v; Augustinus Ritius, De motu octavae sphaerae [...] Item ejusdem Epistola de astronomie auctoribus, in aedibus Joannis de Ferrariis, Tridini 1513; LAZARELUs, Lodouici Lazareli Poete Christiani ad Ferdinandum Regem dialogus cui titulus Crater Hermetis, in Contenta in hoc volumine, fol. 60v-81v; SYMPHORIANUS CHAMPERIUS, Commentarium in Asclepij Mercurij Trismegisti discipuli diffinitiones theologicas ad Ammonem regem, ed. Cesare VASOLI, in ENRICo CASTelli (ed.), Umanesimo ed Esoterismo. Atti del 5. Convegno internazionale di studi umanistici, Oberhofen, 16-17 settembre 1960, CEDAM, Padova 1960 [Archivio di filosofia: organo della Società filosofica italiana, 2/3 (1960)], p. 260-289; PerRone Compagni, « Ermetismo e Cristianesimo », p. 35-36. 
sacerdote, il massimo e gloriosissimo re. Per questa ragione il popolo, ammirando la sua virtù, gli consacrò onori divini. ${ }^{22}$

Il sapiente ermetico, considerato espressione armonica del mago sacerdote, profeta e legislatore, offriva quindi ai Cristiani un esempio reverendo ed encomiabile, oltreché accreditato per la sua vetustà e consonanza col messaggio di Cristo, a cui attingere nell'ottica di formare un teologo cristiano, cioè un interprete meritevole e autorevole della Parola di Dio.

22 AgRIPPA, Oratio habita Papiae, p. 123-124: « Primus qui omnem sapientiae semitam perscrutatus, oratione licet modica, immensa tamen sententiis, de vera sapientia scripsit. Primus omnium de majestate Dei, de ordine spirituum, de animarum naturis sapientissime disseruit. Primus itaque theologiae appellatus est autor et inventor; ferturque universalia sacrorum complexus, conscripsisse viginti sex milia, quingenta et viginti quinque librorum volumina, in quibus admiranda arcana, secretissimique mysteria ac stupenda pandit oracula; non enim ut Philosophus duntaxat locutus est, verum etiam ut propheta multa praesagivit. Nam priscae religionis ruinam, novae fidei ortum, Christi adventum, futurum judicium, mortuorum resurrectionem, renovationem saeculi, beatorum gloriam, peccatorum tormenta praevidit atque praedixit. [...] Hic itaque Mercurius noster, ut acumine intelligentiae philosophos omnes excessit, ita sacerdos perinde constitutus sanctimonia vitae, divinorumque cultu universis sacerdotibus praestitit; unde in tanta hominum veneratione habitus est, ut post Osyridem ab Aegyptiis rex appellaretur. [...] Regiam itaque dignitatem adeptus Mercurius, consitutione legum, administratione regni ac gestorum magnitudine, caeterorum regum gloriam obscuravit, unde merito Trismegistus, hoc est, ter maximus nuncupatus est, quoniam maximus et sapientissimus philosophus, maximus ac religiosissimus sacerdos, maximus ac gloriosissimus rex extiterit, quapropter pro suae virtutis admiratione divinos honores populus illi consecravit». Sulla genealogia di Ermete che Agrippa propone in versione aggiornata cf. PerRone Compagni, Ermetismo e Cristianesimo, p. 35-36: «Sebbene fosse l'Argumentum di Ficino a fornire lo schema di tale biografia, Agrippa se ne distaccava in un punto non secondario. La genealogia ficiniana, affidandosi alla testimonianza di Agostino, faceva risalire il Trismegisto alla stirpe dell'astrologo Atlante, contemporaneo di Mosè: la collocazione di Ermete quattro generazioni più tardi rispetto alla rivelazione di Dio a Mosè permetteva di giustificare le profezie pagane di Ermete e la loro concordanza con la religione ebraico-cristiana entro la tradizionale spiegazione agostiniana della iniusta possessio. Il Crater Hermetis di Lazzarelli aveva invece rovesciato la cauta ricostruzione ficiniana, proponendo l'audace coniectura [...] che all'egizio Ermete dovesse essere fatta risalire l'origine della vera sapienza, da lui poi trasmessa agli Ebrei. Anche Agrippa datava la vita di Ermete a un tempo precedente Mosè; ma ne faceva un ebreo: egli era Enoch, figlio di uno dei molti figli che Abramo aveva generato dalle sue concubine dopo la morte di Sara. Nel riportare il personaggio e il suo insegnamento a una originaria appartenenza al popolo ebraico, Agrippa rendeva omaggio ad Agostino Ricci, che nella Epistola de astronomiae auctoribus aveva appunto sostenuto tale genealogia. [...] Ermete, ebreo, ma vissuto prima di Mosè, si situava così nel tempo indistinto tra prima e seconda manifestazione di Dio, ma era tacitamente innalzato al rango dei grandi patriarchi biblici ». 
Se si considera in un quadro strettamente ideologico la restaurazione della magia assieme al recupero della devozione ermetica, l'operazione di Agrippa esprime attivamente la necessità di liberare le Sacre Scritture dal peso della tradizione scolastica, mirando a ridisegnare in forma calibrata i contorni mutevoli dell'ortodossia. Ai teologi contemporanei di formazione scolastica il filosofo imputa la responsabilità culturale e morale di aver frainteso e corrotto il nucleo originario di verità contenuto nelle Sacre Scritture. Costoro, per mezzo di un impiego indisciplinato della ragione umana, si barricano dietro le sottigliezze del pensiero aristotelico per sezionare, glossare, manipolare e adulterare il testo sacro, risultando così del tutto incapaci di cogliere, al di là della lettera e dei pregiudizi che su di essa erigono, il significato della Parola: quel messaggio recondito che Dio stesso ha rivelato a tutti, ma al contempo riservato in forma autentica e più elevata soltanto agli uomini che, per il tramite della fede e della sapienza, si siano resi meritevoli di riceverlo. ${ }^{23}$

Nel De triplici ratione cognoscendi Deum, risalente al 1516 ma consegnato all'editore solo nel 1529, Agrippa sarà pugnacemente impegnato a ripensare il concetto di teologia, rimarcando l'incompatibilità della professione teologica, genuinamente concepita ed esercitata, con l'attività pseudoscientifica che i dottori scolastici praticano correntemente nelle università. ${ }^{24}$ Tale

23 EAD., «Riforma della magia», p. 122: «Restaurare la magia nel suo primitivo significato di 'sapientia' religiosa - strumento di inveramento della scienza in quanto coincidente col contenuto della rivelazione divina - impone preliminarmente una presa di posizione nei confronti della scienza contemporanea. Bisogna indicare le contraddizioni, l'inutilità pratica e soprattutto i disastrosi effetti morali di una ragione che si è allontanata dalla fonte originaria della sapienza, che ha voluto procedere autonomamente, che crede di poter afferrare persino Dio con i suoi sillogismi: una ragione la cui immagine speculare è la società contemporanea, fatua, immorale, arrogante, lontana dalla erasmiana 'simplicitas' di chi si predispone, fiducioso ma 'attivo', alla rivelazione divina, dispensatrice di una sapienza che diviene anche potenza, possibilità di pratica trasformazione $»$.

24 EAD., «Della vanità delle scienze: note di lettura», Bruniana \& Campanelliana, 11 (2005), p. 131: « Qui [scil. nel De triplici ratione] Agrippa delineava le tre strade per giungere alla conoscenza di Dio, collocandole in una scala di progressiva perfezione e compiutezza: la scienza pagana, che muove dalle creature al Creatore; la cabala ebraica, che conosce Dio attraverso l'interpretazione della Legge e con il ministero degli angeli; infine la vera conoscenza attraverso Cristo nel libro del Vangelo, luce piena del meriggio che dirada la caligine del libro delle creature e rischiara l'umbratilità del libro della Legge. Questa successione cronologica nel manifestarsi di Dio all'uomo contiene l'abbozzo di una serie di princìpi che costituiranno più tardi il nucleo del credo religioso di Agrippa, equamente lontano da ogni chiesa magisteriale, da Roma come dalla Riforma: l'evento della redenzione è una svolta provvidenziale che ha cancellato una volta per tutte la condizione storica dell'uomo macchiato dal peccato originale, riconducendolo alla perfezione del suo momento iniziale e restituendogli la pienezza di ragione e volontà - il libero arbitrio risanato; Dio ha predestinato universalmente gli uomini; nell'individuo risiede la 
differenziazione si appella alla distinzione tra i due livelli di approccio al dettato scritturistico, letterale e allegorico, legittimati a partire dalla complessità del messaggio biblico, la cui comprensione certamente è stata destinata dalla divinità a tutti gli uomini, ma è stata riservata in un modo agli imbecilliores, in un altro ai perfecti. La confusione tra i due livelli, nonché l'impossibilità di sciogliere nelle sue verità l'impianto metaforico-spirituale delle Scritture, attesta per Agrippa non solo l'incompetenza metodologica dei teologi scolastici, bensì soprattutto la crisi teorica nella quale si è arenata la teologia contemporanea, incapace di identificare il proprio oggetto di studio, di insegnarlo correttamente, di predicarlo nella devozione autentica, nonché di esercitare nel nome di Cristo, al pari degli Apostoli, virtù profetiche o facoltà miracolose:

Infatti anche il Vangelo, come la legge mosaica, presenta un significato superficiale, destinato alle persone più deboli, e un significato interno, che è stato rivelato separatamente ai perfetti - nel senso spiegato da Paolo agli Ebrei, [...] quando dice: 'Se volete essere condotti alla dottrina perfetta di Cristo, dovete lasciare l'insegnamento iniziale, nel quale cioè si trattano i princìpi e i fondamenti della sapienza divina che riguardano la penitenza dalle opere morte, il battesimo, i sacramenti, l'imposizione delle mani e l'autorità di assoluzione, la resurrezione dei morti e il giudizio eterno' e gli altri argomenti analoghi, che sono tutti contenuti nell'involucro del Vangelo e sono trattati nelle aule dai teologi scolastici e sono ridotti in problemi da soppesare e da discutere. Le altre conoscenze che invece attengono alla sapienza più alta e alla dottrina perfetta - cioè quale sia 'il dono celeste' e 'la manna riposta, che nessuno conosce se non colui che la riceve', e quale sia la buona parola di Dio, migliore di quella che è trasmessa al volgo in parabole, e quale sia 'il mistero del regno di Dio, che è dato conoscere solo ai discepoli più fidati', e quali le virtù del tempo futuro, quale l'origine e il fine dell'anima e le funzioni degli spiriti angelici, quale la condizione e la qualità di quell'immensa gloria e felicità che attendiamo, [...] - tutte queste conoscenze sono contenute all'interno del Vangelo e nel suo nucleo e sono note soltanto ai perfetti: a loro è stata elargita la scienza delle potenze e delle virtù, dei miracoli e della profezia e di ciò che gli uomini non possono indagare con le proprie forze, a meno che non si siano assoggettati alla virtù dello Spirito santo. ${ }^{25}$

responsabilità di aderire o meno all'offerta divina e quindi la sua salvezza o la sua condanna eterna; la fede si effonde in doni di grazia: non soltanto in buone opere, ma anche e soprattutto in miracoli. La rigenerazione apre la via verso la perfezione, fino al compenetramento con la divinità: ci si fa figli di Dio, ci si trasforma in Colui di cui siamo immagine ».

25 Agrippa, De triplici ratione, p. 148-150: " Habet enim etiam Evangelium, quemadmodum lex mosaica, aliud in cortice propositum imbecillioribus, aliud in medulla, quod segregatim revelatum est perfectis [I Cor. 2, 6-7], sicut de illis loquitur Paulus ad Hebraeos [...] inquiens: 'Si ferri vultis ad perfectam Christi doctrinam, omittendus est sermo incohationis, in quo videlicet tractatur de principiis et fundamentis divinae sapientiae quae sunt de poenitentia ab operibus mortuis, de baptismate, de sacramentis, de impositione manuum et de auctoritate absolvendi, de resurrectione mortuorum et iudicii aeternio' et eiusmodi, quae omnia habentur in cortice 
Ancor prima di formulare questa distinzione fondamentale, Agrippa ritiene urgente denunciare l'imperizia dottrinale e la corruzione morale dei 'Magistri nostri', i quali mancano della 'intelligentia Scripturarum'. Imbevuti del lessico teorico di Aristotele e di Averroè, irrobustiti poi nelle pieghe del frasario scotista e tomista, essi ignorano le bonae litterae, ossia l'educazione letteraria imprescindibile per accostarsi a una testualità complessa, storicamente stratificata e linguisticamente composita, come quella delle Sacre Scritture. ${ }^{26} \mathrm{Al}$ contrario, servendosi artificiosamente della dialettica e della logica per decifrare i misteri divini, gli Scolastici sobillano la ragione umana a scapito di una fede sincera e incontaminata, ferma nella ricezione del messaggio di Cristo; dedicandosi soltanto a chiosare, postillare e commentare senza fine singoli passi della Scrittura, promuovono sterili controversie in campo dottrinale, allontanando i Cristiani dalla verità del Vangelo:

Ma ci sono alcuni altri che parlano con le lingue, gonfi di scienza umana; che, anzi, non arrossiscono nel pronunciare le loro menzogne su Dio con la vita e con la lingua; che con lo spirito impudentemente piegano tutta la Scrittura alle proprie bugie; che misurano i misteri divini con il metodo della ragione umana; che, corrompendo la parola di Dio, con sacrileghe glosse tratte fuori dalle loro teste, stabiliscono le loro invenzioni fantastiche; che, come ladri e furfanti, follemente si

Evangelii et in scholis tractantur a scholasticis theologis et in problemata disputanda et discutienda deducuntur. Illa autem pertinentia ad meliorem sapientiam et perfectam doctrinam - videlicet quod sit 'donum coeleste' et 'manna absconditum, quod nemo scit nisi qui accipit', et quod sit bonum Dei verbum, melius illo quod foris vulgo traditur in parabolis, quodque 'mysterium regni Dei datum nosse solis secretioribusp discipulis', et quae virtutes saeculi futuri, quae origo et finis animae et ministeria angelicorum spirituumq, quae conditio et qualitas illius immensae gloriae et felicitatis quam expectamus, quam 'nec oculus vidit, nec auris audivit, nec in cor hominis ascendit' - haec omnia continentur in medulla et nucleo Evangelii et non nisi perfectioribus cognita sunt; quibus data est scientia potestatum et virtutum, miraculorum et prophetiae et cetera, quae homines propriis viribus indagare non possunt, nisi qui subiecti fuerint virtuti Spiritus sancti [Georgius, Harm. mun., II, 5, 9, fol. 275r] ». La traduzione dei passi tratti dal De triplici ratione è di Perrone Compagni. Come rileva la studiosa, il passo riportato, originariamente assente nella stesura manoscritta del De triplici ratione, costituisce un'interessante integrazione testuale dovuta alla lettura del De harmonia mundi di Francesco Zorzi, pubblicato a Venezia nel 1525. Il brano fu dunque inserito nel corso della revisione a cui Agrippa sottopose l'opuscolo poco tempo prima di stamparlo ad Anversa nel 1529; cf. PERRONE CoMPAGNI, Ermetismo e Cristianesimo, p. 74-75.

26 Cf. AgripPa, Defensio propositionum, fol. Cv: «Quod ut melius efficiam, nescio an modo mihi necessarium sit supercilium, nasusque theologicus et obvinctum tot fasciis caput, quodque magistrorum nostrorum, ut vocatis, glossas, postillas, conbiblicationes, resumptiones, reportata, pariter atque pugnantia invicem vocabula, nominalium realium, primarum ac secundarum, inventionum, propriarum et impropriarum, implicitorum et explicitorum sensuum, et reliquas scholasticarum nugarum voces, scotistarum vel thomistarum spiritu aliquando fuerim edoctus $»$. 
arrogano il santo titolo di teologi, mentre la loro unica fatica si riduce a contese e risse verbali. ${ }^{27}$

Arrogandosi il possesso della tradizione e il sigillo dell'ortodossia, essi screditano gli interpreti più validi e riconosciuti dalla tradizione, diffondono interpretazioni erronee o storie inventate, alimentando così i conflitti e l'eresia nella comunità dei fedeli. Se posti a confronto con i Padri della Chiesta, gli Scolastici si dimostrano pericolosamente impreparati a livello dottrinale, poiché ricercano una conferma delle verità di fede in luoghi diversi dalle Scritture, come i testi di Aristotele o di altri filosofi pagani, trascurando così il Vangelo, la fonte primaria, indispensabile anche soltanto per avviare un discorso teologico legittimo. ${ }^{28} \mathrm{Sul}$ piano strettamente esegetico sono sprovvisti di un metodo rigorosamente fondato: non sanno decodificare le numerose oscurità della narrazione biblica collazionando, ad esempio, passi simili delle Scritture al fine di individuare gli absconditi sensus del Verbo di Dio. Senza nemmeno onorare umilmente l'autorità degli uomini santi che li hanno preceduti, offrendo nelle opere e nelle parole testimonianza della vera fede, rigettano la lezione degli antichi dottori, i quali,

27 ID., De triplici ratione, p. 154: «Sed sunt quidam alii, qui linguis loquuntur, humanis scientiis inflati, imo qui vita et lingua de Deo mentiri non erubescunt, qui suo spiritu omnem Scripturam ad sua mendacia impudentissime torquent ac mysteria divina ad humanae rationis methodum exigunt inventisque e capitibus suis glossis sacrilegis, adulterato verbo Dei [II Cor. 2, 17], sua portenta stabiliunt ac a sanctum theologiae nomen furto et rapina sibi temere usurpant solisque operam dant contentionibus et rixosis disputationibus ".

28 Questo principio metodologico sarà ribadito anche in un opuscolo apparentemente controverso, nel quale Agrippa si interroga sul valore della Ethnicorum philosophia per la formazione di un cristiano, specialmente di un teologo. Si tratta della Dehortatio gentilis theologiae, la cui redazione originaria risale all'incirca alla fine del soggiorno in Italia per poi essere rielaborata nel 1526. Scritta su invito di alcuni sodali ecclesiastici, appassionati lettori del Corpus Hermeticum e dei filosofi neoplatonici cari ad Agrippa (Plotino, Porfirio, Giamblico, Proclo), l'orazione dissuasoria si presenta come un'invettiva contro la letteratura pagana, pericolosamente responsabile di sviare i Cristiani dalla dottrina del Vangelo, fonte esclusiva della verità teologica. Nonostante l'opuscolo sembri confutare apertamente l'impostazione umanistica caldeggiata da Agrippa, l'oratore chiude il suo discorso ribaltando la condanna appena espressa e rivalutando completamente la letteratura pagana. La sapienza dei Gentili, a parere di Agrippa, non costituisce pericolo per il buon cristiano e, conseguentemente, non merita il suo disprezzo, poiché contiene insegnamenti conformi alla dottrina di Cristo. Il cristiano potrà accogliere e persino professare apertamente alcune dottrine filosofiche dei Pagani, ma solo nel caso in cui egli abbia saldamente interiorizzato e irrobustito nella propria coscienza il messaggio evangelico: cf. AGRIPPA, Dehortatio gentilis theologiae, in ID., De Nobilitate \& Praecellentia Foeminei sexus, ad Margaretam Augustam Austriaco(rum) et Burgundionum Principem..., Michael Hillenius in Rapo, Antverpiae 1529, fol. Iv-I2r: « Nam ad divinarum rerum veridicam cognitionem puro animo opus est et non infecto pravis opinionibus. [...] Igitur in theologico studio nihil vobis prius esse debet quam evangelica doctrina [2 Th., 2, 14]»; Ibid., fol. H6r: «Quicunque enim Dei notitiam aliunde quam ab Evangelio parandam putant, ii vere sunt qui Spiritum sanctum incessunt; quae blasphemia neque in hoc neque in futuro saeculo remittitur ». 
sostenuti dallo Spirito divino, fornirono con esposizioni e commentari una via privilegiata per accedere alle verità rivelate..$^{29} \mathrm{~L}$ 'arroganza di cui essi si rendono espressione fomenta così la loro illusione di poter «tutto saggiare, affrontare, risolvere e interpretare ", ossia ricondurre i più alti enigmi della religione ai fantasmi, alle illusioni e persuasioni della ragione umana: ${ }^{30}$

E, a questo punto, tutto ciò che nella fede e nella religione è semplice, schietto e puro costoro hanno reso molteplice, oscuro e sudicio. Con le loro assurde schermaglie e la loro futile loquacità hanno sfigurato, perturbato, contaminato tutta la teologia; e hanno fabbricato non una teologia divina e neppure umana, bensì una qualche loro, non dirò certo 'teologia', ma una rozza, odiosa, capziosa $\mathrm{e}$ diabolica vanità, una rapsodia di umane opinioni e di frottole filosofiche. ${ }^{31}$

La presunzione dei dottori scolastici non si limita solo al campo dottrinale, ma si ripercuote inevitabilmente sulla condotta pubblica. La comunità dei fedeli scopre in essi la miseria morale di uomini soggiogati al vigore durevole delle passioni, $\mathrm{i}$ quali esibiscono uno stile di vita segnato a tal punto dal vizio e dall'avidità di potere, da risultare indegno per un teologo di professione. Denunciare la corruzione morale e spirituale delle figure incaricate di formare le coscienze costituisce una rivendicazione costante nel programma culturale di Agrippa. ${ }^{32} \mathrm{La}$

29 Sotto questo profilo l'approccio di Agrippa alla letteratura patristica, al di là dell'influsso di Erasmo, è paragonabile a quello di Lorenzo Valla, cf. CRISTOPHER S. CELENZA, Il Rinascimento perduto. La letteratura latina nella cultura italiana del Quattrocento, Carocci, Roma 2004 (Lingue e letterature Carocci, 176), p. 187: «Valla, come tutti gli umanisti, era profondamente immerso negli autori classici non cristiani, ma l'antichità cristiana aveva per lui un significato speciale, poiché la lingua dei Padri della Chiesa combinava la naturale eleganza delle antiche forme di espressione con l'umiltà adatta a un vero cristiano ».

30 AgRIPPA, De triplici ratione, p. 164: « Tunc freti sophistica sua insolentia, omnia se posse attentare, aggredi, dissolvere et interpretari putant ».

31 Ibid., p. 164: «Tunc irruentes suis ineptiis, inquinamentis et blacteramentis rixosisque disputationibus, ad quod artificium iam linguas armatas habent, omnia quae in fide et religione simplicia, sincera et pura sunt [ReuchuIN, Verb. Mir. III, fol. g5v], multiplicia, caliginosa et sordida reddiderunt omnemque theologiam suis absurdis altercationibus ac futili verbositate confuderunt, conturbarunt, polluerunt inveneruntque non divinam nec humanam quidem, sed nescio quam suam, non dico theologiam, sed squalidam, odiosam, cavillatoriam et diabolicam vanitatem, humanarum opinionum philosophicarumque nugarum rhapsodiam [REUCHLIN, Verb. mir. II, fol. d1r]».

32 Ancora molti anni più tardi, nella sferzante Apologia scritta contro le violente censure pronunciate dei teologi di Lovanio e della Sorbona, a fronte dello scandalo suscitato dalla pubblicazione De incertitudine et vanitate scientiarum nel 1530, Agrippa fornirà un ritratto eloquentemente mordace dei suoi oppositori: cf. ID., Apologia aduersus calumnias, cap. 7, fol. D2r: "An soli magistri nostri et Scholastici sophistae homines sunt et spirituales, caeteri omnes stipites sunt, aut fungi, aut asini, quicunque non sapiunt quod ipsi sapiunt? Paulus vocat animales homines [I Cor., 2, 14], qui ducuntur humanis affectibus, unde Iacobus appellat animalem sapientiam [Iac., 3, 15], quae tumet humanarum rationum et syllogismorum fiducia, 
scienza ingannatrice che essi professano, alimentandosi della contesa e della prepotenza di una ragione indomita, ossia non sottoposta al vaglio dell'umiltà $\mathrm{e}$ della pietà, rende il loro intelletto superbo e aggressivo, proteso ad avvelenare l'anima nella polemica religiosa: « Difatti per l'anima non c'è peste maggiore che il ragionamento, la discussione, la contesa sul divino, perché annientano la ragione, corrompono l'intelletto, uccidono la fede ${ }^{3 .}{ }^{33}$ Per questa via si estingue durevolmente la purezza di una devozione vissuta, nella preghiera, nei digiuni, nella predicazione, vale a dire nel culto che dovrebbe animare l'assiduo contatto con la Parola di Dio. Come potranno mai costoro afferrare la verità di Cristo, edificare nella fede la comunità dei fedeli, amministrare con giustizia la Chiesa universale, o persino operare miracoli sull'esempio degli Apostoli e dei Profeti, se difettano a tal punto dello spirito divino? ${ }^{34}$ In quanto tali, questi falsi sapienti, cui è affidato un compito delicatissimo per il consorzio civile, esprimono l'archetipo di un'umanità indifferente, ostile alla religione, dimentica di Dio. ${ }^{35}$

carens Spiritu Dei, quales sunt qui pro suo affectu citant et torquent, et interpretantur et applicant Scripturas ex humanis ratiunculis et philosophia Gentium, quaerentes quae suae sunt, non quae sunt Dei. Non omnes ergo Magistri nostri, non omnes caputiati et cucullati spirituales sunt, si vitiorum quorumlibet genere foedati, superba hypocrisi, insatiabili avaritia, impudenti calumnia, odio, ira, invidia et tecta cuculla luxuria, postergata imitatione Christi, affectiones sequuntur animales, quantacunque licet contentiosa loquacitate praediti sint et humanarum scientiarum opinionibus inflati, sed qui ad Sacras Scripturas, etiam vitae, morum ac fidei rationes commodant ».

33 ID., De triplici ratione, p. 160: «Nulla enim maior pestis animae quam ratiocinatio, quam altercatio, quam disputatio de divinis, quae evertit rationem, pervertit intellectum, deiicit fidem »; cf. ID., Dehortatio gentilis theologiae, fol. I2r: "Curiosas autem de Deo divinisque inquisitiones quaestionumque altercationes et disputationum pugnas, iuxta Apostoli praeceptum [Tit., 3, 9; 2 Tim., 2, 23-26], omnino vitate ».

34 Sulla scia della critica di Reuchlin alla teologia contemporanea, anche Agrippa certifica nell'incapacità dei sacerdoti e dei prelati di operare miracoli la separazione materiale e spirituale della Chiesa attuale dallo Spirito di Cristo; cf. NAUERT, Agrippa and the Crisis, p. 48-49: «Agrippa's belief that the truly illuminated soul could perform works of magic which seemed miraculous even led him into an attack on the corruption of the church in his own day. The early church, he argued, had been aided by the miraculous works of the apostles and of their immediate successors, who were able to perform these miracles precisely because they did possess the pure and spiritual knowledge of the revelation contained in the Bible. Properly, Agrippa felt, the prelates of the church ought always to be gifted with such wonder-working powers based on true knowledge. If present-day theologians and prelates were unable to perform any such miracles (and they were unable), that inability was merely further proof of their corruption and lack of faith. Their error was that they founded their knowledge on reason instead of on faith. They were adulterating the Word of God with their own attempts to bend it to the demands of human reason ».

35 È quanto Agrippa ribadisce a più riprese anche altrove; cf. ad esempio Defensio propositionum, fol. G5v: «Sed quod est istud novum hominum, ne dicam haereticorum, genus, qui, ut inquit Paulus, non propter bonam voluntatem, sed propter contentionem Christum praedicant; qui evacuantes crucem Christi, sacratissimam theologiam, quae nullam sub artem cadere potest, persuasibilibus humanae sapientiae verbis ac logicis regulis astringunt; qui ea, quae nisi sola 
A partire da una diagnosi così articolata, ripensare l'approccio al testo sacro, depositario dell'unica sapienza salvifica, ricercando con strumenti critici la purezza del significato originario, esprime in Agrippa la volontà di ridefinire il concetto di teologia. ${ }^{36}$ La teologia, in quanto indagine sul significato della Parola, dovrà abbandonare i sofismi e le controversie dialettiche, dovrà rigettare i metodi in uso nelle aule universitarie, per tradursi in una meditazione profonda e responsabile sul messaggio cristiano. ${ }^{37}$ Ricercare il senso autentico, non adulterato o travisato, delle Scritture significherà allora disciplinare l'attività della ragione, confortarla con il sostegno della fede e della Grazia elargite da Cristo, affinché l'anima dell'uomo si elevi, nel silenzio delle apparenze sensibili, a contemplare le verità della mente e i misteri divini in cui è descritto il sentiero di salvezza. Solo in questo modo l'esercizio della teologia diviene per il sapiente rinascita spirituale nella contemplazione di Dio, addestramento ad un esercizio corretto della facoltà razionale, catarsi morale dell'intera persona. ${ }^{38}$

fide creduntur, suis syllogismis sese invenire ac demonstrare posse praesumunt; qui monstricos casus excogitantes, novasque chimeras quotidie adinvenientes, Deo et omni creaturae illudunt ».

36 PeRrone Compagni, Della vanità delle scienze, p. 130: « Nel condurre la sua guerra contro i 'temerari giganti et inimici delle sacre Lettere', Agrippa persegue anche un obiettivo di riforma gnoseologica e morale; ma ha in mente qualcosa di molto più ampio, un programma religioso e concretamente politico. La filosofia si fa progetto di rinnovamento spirituale e istituzionale, auspicando una revisione radicale non solo delle forme esteriori della religione e dei modi del suo insegnamento, bensì dello stesso concetto di teologia, cioè dello stesso fondamento su cui si basa l'ordine gerarchico e sacramentale della Chiesa. Si tratta di una contrapposizione che è ideologica prima ancora che dottrinale, di una lotta per l'egemonia culturale prima ancora che di un confronto tra ortodossia ed eterodossia. Agrippa non conduce una guerra 'ideale', di scuola, contro il passato e la tradizione; combatte invece un duello reale e personale contro la corruzione del presente, contro i filosofi, i teologi, i religiosi del proprio tempo ».

37 NAUERT, Agrippa and the Crisis, p. 50: « Like the other humanists, he [scil. Agrippa] upheld the ideal of a return to the pure, undefiled faith of the early church, in opposition to the contentious wrangling of scholastic theology. To the proud rationalism of the professional theologians, Agrippa and his friends contrasted the firm and humble faith of the true believer, who praises God for illuminating man by grace, and who prepares himself for enlightenment with vigils, fasts and a life lived in imitation of Christ. Only to such persons, Agrippa felt, will God grant knowledge which is beyond the reach of human investigation, the knowledge of the true mysteries of religion, which are contained not in the external revelation, but in its inner meaning ».

38 Perrone Compagni, Della vanità delle scienze, p. 131: «Attraverso una critica moralistica della corruzione delle persone che praticano la scienza, Agrippa pone sotto accusa l'inadeguatezza delle strutture a cui è affidata la direzione delle coscienze; ma ne mette anche in discussione la preminenza (la teologia è affare di tutti) e financo la competenza, perché il vero teologo è colui che è guidato dallo Spirito, colui che pratica il suo cristianesimo come un esercizio di 
Ogni tensione d'amore per la sapienza viene dallo Spirito santo mediante il nostro signore Gesù Cristo. La vera sapienza è conoscenza di Dio, illuminazione della mente, riforma della volontà e desiderio di una ragione retta, quasi una salda legge di vita, che rende santa l'anima dell'uomo, adegua la sua esistenza a Dio, gli indica cosa egli debba fare e cosa rifuggire: con termine diverso noi chiamiamo questa sapienza 'teologia'. Tale sapienza e vera conoscenza di Dio - anzi, tale sorta di contatto essenziale con Dio, più eccellente della conoscenza - è divinamente trasmessa nel Vangelo. Infatti né Dio può essere veramente conosciuto senza il Vangelo, né il Vangelo può essere veramente compreso senza la grazia divina. È senza dubbio evidente che gli insegnamenti di Dio non possono essere penetrati se non mediante Dio, come dice il Profeta: 'Nella tua luce vedremo la luce'. Mercurio Trismegisto chiama questa luce 'mente della essenza divina', luce stessa che scaturisce da Dio. ${ }^{39}$

La professione teologica coincide con la scelta di abbracciare un percorso di perfezionamento umano radicato nell'esegesi scritturistica, fondato sulla dottrina del Vangelo e realizzabile grazie all'apprendimento della 'luce di Dio', cioè della vera sapienza. Accogliere gli insegnamenti divini significa tradurre in prassi concreta l'esperienza religiosa, orientando attivamente la vita individuale all'insegna dell'obbedienza alla Legge, secondo la quale al cristiano è prescritto di amare Dio e di amare il prossimo, ossia di custodire la devozione e di praticare la giustizia. Questo insegnamento è tramandato unanimemente nelle parole di Cristo e in quelle di Ermete Trismegisto:

Ma invano conosciamo Dio, se non lo onoriamo convenientemente e se non viviamo con gli uomini secondo la legge. Dice pertanto Ermete: 'La sfida della devozione religiosa consiste nel riconoscere Dio e nel non recare offesa a nessuno'. È lo stesso precetto che ha dato Cristo, quando dice: 'Ama il signore Dio tuo e il

perfezionamento e di santificazione, attraverso il superamento di sé stesso, dominando le apparenze e disprezzando i valori mondani : il vero teologo è il rigenerato in Cristo ».

39 AGRIPPA, De triplici ratione, p. 134: «Omne studium amorque sapientiae ex Spiritu sancto est per dominum nostrum Iesum Christum. Ipsa vera sapientia Dei cognitio est, illustratio mentis, voluntatis correctio appetitioque rectae rationis, quaedam vitae certa lex, sanctificans animam hominis, Deo disponens vitam, quid agendum, quid omittendum demonstrans; quam nos sapientiam alio vocabulo theologiam vocamus. Haec sapientia veraque Dei cognitio, imo contactus quidam Dei essentialis, melior quam cognitio, traditur divinitus in Evangelio. Neque enim Deus ipse sine Evangelio vere cognoscitur, neque $c$ Evangelium absque divina gratia vere intelligitur. Manifestum enim est ea, quae ex Deo tradita sunt, non nisi ex Deo intelligi posse, sicut ait Propheta: 'In lumine tuo videbimus lumen" [Ps. 35 (36), 10]. Quam lucem Trismegistus Mercurius mentem vocat divinae essentiae, lucem ipsam exorientem Deo»; cf. Mercurii Trismegisti Pimander. Liber de sapientia et potestate Dei, I, in Contenta in hoc volumine, fol. 4rv: «Sum, inquit ille, Pimander, mens divinae potentiae [...] Lumen illud ego sum, mens deus tuus [...] Mentis vero germen lucens Dei filium [Corpus Hermeticum. Asclepius, ed. ARTHUR D. NocK, ANDRÉ M. J. Festugière, Les Belles Lettres, Paris 1945, vol. I, p. 7-8] ». 
prossimo tuo come te stesso'. Questi due comandamenti sono necessari alla salvezza e sono la scaturigine di ogni bene: il primo è fonte di devozione, il secondo di giustizia. ${ }^{40}$

Il vero teologo, dunque, in quanto degno interprete delle Scritture, sarà esempio costante di carità verso il prossimo, non esposizione della superbia umana; sarà modello vivente di integrità morale, non esemplare di avida insolenza e litigiosa ambizione; secondo la lezione trasmessa dai Profeti e dagli Apostoli, egli sarà custode appassionato e diligente di una fede saldissima in Dio.

Riformare la teologia richiede necessariamente una trasformazione dell'impianto metodologico, ossia dei fondamenti del discorso ermeneutico. Il progetto di Agrippa muove dalla volontà di sostituire all'esegesi scolastica un nuovo metodo di approccio alle Scritture, individuato recuperando da un lato la più recente filologia biblica, di cui Erasmo e Lefèvre d'Étaples avevano offerto testimonianza esemplare, e dall'altro il modello sapienziale di Reuchlin, ossia un'esperienza religiosa che, tramite l'esegesi della Parola di Dio, rigeneri l'uomo per renderlo operatore di miracoli. ${ }^{41}$ Così concepito, l'approccio umanistico di Agrippa si richiama ad alcuni parametri fondamentali che, sebbene parzialmente enunciati nel periodo italiano, saranno sviluppati e collaudati successivamente nelle opere della maturità.

In primo luogo, a scapito dei rencetiores scriptores, ossia della tradizione scolastica medievale, che con le sue polemiche e faziosità ha stravolto alla radice il prezioso insegnamento dei suoi più illustri iniziatori, come Tommaso d'Aquino e Alberto Magno - i quali, d'altronde, si servivano delle dottrine dei Peripatetici per combattere le eresie nella Chiesa, non per alimentare la propria vanagloria Agrippa sottolinea il valore della teologia dei Padri - Dionigi, Lattanzio, Origene, Girolamo, Eusebio, Agostino, per ricordarne alcuni - a cui attribuisce indiscussa preminenza:

40 Agrippa, De triplici ratione, p. 116-118: « Sed frustra cognoscimus Deum, nisi illum rite colamus et legitime cum hominibus vivamus. Unde inquit Hermes: 'Certamen religiosae pietatis est recognoscere Deum, iniuriam inferre nemini' [Corpus Hermeticum. Pimander, X, fol. 23v (NockFestugière, 1, p. 122)]. Quod etiam praecipit Christus, dicens: 'Dilige dominum Deum tuum et proximum tuum sicut te ipsum' [Mt. 22, 37-39; Mc. 12, 30-31; Lc. 10, 27]. Haec duo praecepta ad salutem necessaria sunt suntque fons omnis boni: horum primum pietatis, alterum iustitiae est ».

41 Perrone Compagni, Ermetismo e Cristianesimo, p. 16, n. 27: «Anche Reuchlin aveva avvertito la necessità di un sapere che si facesse azione, ars miraculorum; ma, a fronte degli esiti fallimentari della magia tradizionale, il De Verbo mirifico proponeva come alternativa più efficace e sicura la magia cabalistico-cristiana fondata sull'uso del nome di Gesù, verbum mirificum, che sancisce l'unione mistica e concretamente si traduce in una fonte di attività sovrumane operate da Dio attraverso l'uomo rigenerato e 'deificato'. La pratica reuchliniana era dunque conseguenza di una esperienza esclusivamente religiosa e poteva essere definita magica solo per analogia ». 
Nelle loro opere è così isolata, così rara la citazione di testi scritti, laddove ci sia qualcosa degno di essere menzionato; e quando c'è, essa proviene dal Vecchio Testamento, dai Vangeli, dagli Apostoli, da autori più antichi. Sono uomini che non si pavoneggiano, che senza dubbio contano nella sola grazia divina, consapevoli del loro sapere, ottimi maestri, non timorosi del giudizio altrui, veritieri, 'capaci di non guardare in faccia gli uomini'. Costoro ci hanno elargito doni tratti dal loro tesoro, imitando Cristo, che 'come il buon padre di famiglia ha tratto fuori dal suo tesoro beni nuovi e vecchi'; e in ogni cosa con parole e opere hanno prodotto negli uomini il frutto della vera religione e della fede per la salvezza eterna. ${ }^{42}$

Nella riflessione sulle prerogative della verità storica, tale attestazione di autorevolezza assegna agli scrittori più antichi un ruolo privilegiato. A parere di Agrippa essi devono la loro affidabilità ai criteri impiegati per documentare gli eventi narrati. In alcuni casi, avendo partecipato ai fatti in prima persona, gli antiquiores hanno reso sé stessi garanti della veridicità; in altri la prossimità temporale agli eventi descritti ha sottratto incertezza e difficoltà al loro compito; in altri ancora, la credibilità della narrazione è stata irrobustita dal ricorso a fonti accreditate, indispensabili per riferire con accuratezza di eventi lontani nel tempo. ${ }^{43}$ Particolare importanza, tra i criteri di validazione metodologica, è assegnata da Agrippa ai publici notarii, considerati fonti preziosissime per la conoscenza storica. Si tratta in altre parole di sacerdoti che svolgevano la funzione di notai, segretari o archivisti, deputati alla redazione di cronografie, alla compilazione di annali, alla registrazione della storia ecclesiastica presso biblioteche $\mathrm{o}$ archivi pubblici ${ }^{44}$ Tale attività recuperava in ambito cristiano la

42 AGRIPPA, De triplici ratione, p. 170-172: « In quorum [scil. prisci theologi] scriptis tam simplex, tam rara invenitur scripturarum citatio, ubicunque aliquid memorandum est, et illa quidem ex Veteri Testamento, ex Evangeliis, ex Apostolis, ex remotioret antiquitate - nihil se iactantes, homines sane in divina gratia solum confidentes, suae sapientiae conscii, doctores optimi, nullorum iudicium timentes, veraces, 'non respicientes in faciem hominum' [Mt. 22, 16]. Qui ex suis thesauris nobis largiti sunt munera, imitantes Christum, qui 'tanquam bonus paterfamilias de thesauris suis protulit nova et vetera' [Mt. 13, 52], in omnibus verbo et opere fructificantes in hominibus fructum verae religionis et fidei ad salutem aeternam ».

43 Di quest'ultimo criterio è esemplare per Agrippa il caso di Eusebio di Cesarea; cf. AgripPA, Defensio prepositionum, fol. D6r: «Quare in primos fontes, ad vetustissimos scriptores, penes quos est inviolata veritatis fides, semper recurrendum est. Inter quos est Eusebius unus omnium praecipuus, qui omnem historiam suam, ut ipse fatetur, ex Clementi Alexandrino, ex Egesippo, ex Irenaeo, ex Theophilo et aliis plerisque publicis et Apostolorum proximis scriptoribus desumpsit. Ideoque sine controversia in ecclesiastica historia ab omnibus recipitur ».

44 VAN DER Poel, Cornelius Agrippa, p. 89-90: «To illustrate this, Agrippa refers to a Biblical episode: after the Jewish people returned from the exile in Babylon, the controversy concerning the building of the temple was solved as a result of the utilization of the official records of the Persians (Ezra 5-6). Therefore, those authors are the most trustworthy who had such an official function and either personally registered the events or copied them from a primary source. This principle of the trustworthiness of the oldest source available, Agrippa continues, has been held valid throughout the ages, and for this reason the early popes ensured the official 
professione più antica del cancelliere, un segretario incaricato di redigere gli atti di un tribunale per conto dello Stato, oppure, in altri casi, quella dello storiografo di corte - ruolo che lo stesso Agrippa avrebbe rivestito ad Anversa diventando indiciarius e historiographus per conto di Margherita d'Austria nel $1529-{ }^{45}$ ossia uno scrittore di pregio, a cui è demandato il compito di coltivare e trasmettere in nome delle autorità la memoria delle opere compiute dai sovrani. A questo proposito, nel 1534, consegnando all'editore la versione rielaborata della Defensio che accompagnava le De beatissimae Annae monogamia, ac unico puerperio propositiones, un opuscolo teologico risalente al 1519 - dedicato alla controversa questione del trinubium di Sant'Anna - in cui aveva difeso pubblicamente Lefèvre d'Etaples dalle accuse di eresia mosse da Claudio Salini, inquisitore domenicano, ${ }^{46}$ Agrippa avrebbe precisato il suo approccio storiografico:

Per quanto riguarda la verità storica dobbiamo credere indubbiamente agli autori che furono presenti, memori o prossimi ai tempi delle cose narrate, ai luoghi, alle azioni, alle persone, o che attinsero le loro storie a scrittori più antichi di comprovata fede. Infatti, poiché un tempo i sacerdoti erano pubblici notai, incaricati di registrare le storie, le origini, gli annali e i tempi, i loro scritti venivano conservati con valore giuridico negli archivi e nelle biblioteche. [...] Perciò è necessario riporre la massima fiducia negli autori che furono presenti agli eventi in qualità di pubblici notai, o che registrarono con valore giuridico gli eventi attingendo a notai più antichi. Come oggi si definisce pubblico e convalidato un documento che sia stato trasmesso in buona fede da un notaio precedente ad

recording of church history. To exemplify this, Agrippa mentions that Pope Fabian (d. 250) appointed seven 'collaterals' (later to be called cardinals) who were each in charge of a notary whose duty it was to record the deeds of the martyrs. He also mentions that Popes Marcellus and Sylvester I saw to the recording of historical events ».

45 Ibid., p. 41.

46 Sulla disputa cf. NAUERT, Agrippa and the Crisis, p. 61: « To the old legend of St. Anne, who was said to be the mother of the Virgin Mary, medieval tradition had added embellishments, among them the belief that after bearing the Virgin and after the death of her first husband, Joachim, she had married twice more, once to Cleophas and one to Salome, by each of whom she had a daughter. All three daughters; and they married Joseph, Alpheus, and Zebedee, bearing, respectively, Jesus Christ; St. James, the Less, Joseph the Just, Simon, and Jude; and James the Greater and the Apostle John. The attack on this belief came first of all from the greatest figure among French humanists, the linguist, theologian, and philosopher Jacques Lefèvres d'Etaples. Agrippa became involved in this controversy only because he had defended Lefèvre's views in discussion at Metz, [...], and then, [...] had been publicly denounced from the pulpit as a rank heretic. In response to this accusation, Agrippa drew up a brief treatise based on Lefèvre's discussion of the question, The tribus et una. His foes, in turn, composed articles of refutation, to each Agrippa wrote a point-by-point rebuttal. His chief adversaries were Dominicus Delphinus of the observant Franciscan, Nicolaus Orici of the Minorites, and, above all, Claudius Salini, prior of the Dominican friary at Metz and a recent doctor of Paris. All three, be it noted, were members of the regular clergy, wich had already taken up the defense of tradition against the humanists in the notorious Reuchlin controversy earlier in the same decade ». 
un notaio seguente, allo stesso modo anticamente venivano sempre conservati in questa maniera i documenti nelle monarchie dei Persi, dei Medi, degli Assiri, dei Greci e dei Romani. E nella chiesa delle origini, gli Apostoli e i loro successori incaricavano sempre alcuni notai, di autorità pubblica, di redigere con valore giuridico la storia della chiesa e del Vangelo. ${ }^{47}$

D'altro canto è più che necessario respingere le scelte dei moderni teologi, i quali, nella maggior parte dei casi, pretendono di fondare la certezza dei fatti sulle loro convinzioni, disprezzando così le opinioni degli antichi, oppure si ostinano a riportare errori, falsità, inganni pubblicamente accertati ${ }^{48}$ Qualora si decida di attingere comunque alle loro esposizioni, occorrerà verificare che quanto affermato presupponga un metodo rigoroso e una solida preparazione. Nei termini di Agrippa, gli iuniores scriptores, qualora rivendichino l'autorità dottrinale in campo storico-religioso, dovranno dimostrare da un lato una fede incrollabile in Dio, costantemente alimentata nelle parole e nelle azioni, dall'altro il possesso di un'autentica erudizione, che prevede necessariamente uno studio assiduo delle fonti storiche più antiche, nonché la conoscenza delle lingue, delle istituzioni, dei riti, delle tradizioni che animano il panorama culturale delle Scritture. La preparazione teologica richiede persino lo studio della natura, nell'ottica di comprendere adeguatamente i fenomeni fisici descritti in molti luoghi della Bibbia. Una formazione solida e organica costituisce il presupposto necessario per distinguere $\mathrm{i}$ veri interpreti della Parola, che operano

47 AGRIPPA, Defensio propositionum, fol. D5r-v: « De his quae ad historicam veritatem pertinent illis autoribus absque repugnantia credendum est, qui narratorum temporibus, locis gestis, personis fuerunt praesentes, aut vicini, seu memores, vel historiam suam ex antiquioribus probata fide exceperunt. Cum itaque olim sacerdotes essent publici notarii rerum gestarum, originum, annalium et temporum, quorum scripta publica fide in bibliothecis et archivis servabantur. [...] Ideo his maxima fides habenda est, qui fuerunt rerum gestarum praesentes publici notarii, vel qui ex antiquioribus notariis haec publica fide exceperunt. Sicut et hodie publicum et probatum dicitur instrumentum, quod a notario per alium posteriorem notarium bona fide traducitur, ita semper $\mathrm{ab}$ antiquo in monarchiis Persarum, Medorum, Assyriorum, Graecorum et Romanorum, praecipue servatum est. Et in prima ecclesia, ab ipsis Apostolis, eorumque successoribus, semper aliqui publicae autoritatis notarii deputati sunt, qui publica fide Evangelii ecclesiaeque texerent historiam »; cf. VAN DER POEL, Cornelius Agrippa, p. 89-90.

48 AGRIPPA, Defensio propotitionum, fol. D6v-D7r: «Contra nunc iunioribus scriptoribus, quibus haec sunt adventicia, nova et a vetustate nimis remota, in quibus non perseverant priscae literae et scribendi mores, ritus, studia, leges, nisi veterum inviolata testimonia, validissimae rationes, bona fide produxerunt, illorum fidei non satis tuto acceditur et dicta illorum invalida sunt, ut a quibus neque argumentum, neque autoritatis ullum suffragium sumi possit. Similiter qui non secuti doctrinam maiorum, solo auditu, vel populare errores et nullo certo autore apocriphas vel privatas opiniones scribunt, nullo modo sunt recipiendi nisi ubi a publica fide non dissentiunt. Qui vero adeo indociles sunt, quod antiquioribus de antiquitate credere nolunt, sed, contra antiquiorum traditiones, de rebus gestis ac historiis altercando, quae sibi videntur placentve utcunque pertinaciter defendunt, hii eadem facilitate contemnendi sunt qua contendunt »; cf. VAN DER POEL, Cornelius Agrippa, p. 90-91. 
onestamente per avvicinare gli uomini alla dottrina di Cristo, dai cattivi predicatori, i quali, muovendo dalle loro illusioni, menzogne o insidie intrappolano nella gabbia dell'ignoranza o vincolano con la zavorra delle opinioni correnti la comunità inerme dei fedeli. ${ }^{49}$ Di tale preparazione enciclopedica, questa la diagnosi di Agrippa, i più recenti scrittori, principalmente maestri della disputa e del sillogismo, difettano alla radice..$^{50}$

In secondo luogo, la distinzione tra cortex et medulla, ossia tra una considerazione superficiale che si affida, come nel caso dei 'Magistri nostri', alle armi della logica per sciogliere le ambiguità testuali, e un approfondimento spirituale che, erede della distinzione medievale tra un approccio allegorico, morale o anagogico, invoca il soccorso della fede per oltrepassare il dettato scritturistico, rimanda inevitabilmente alla difficoltà strutturale, connessa ai

49 AGRIPPA, Defensio propositionum, fol. E2v-E3v: «Nam qui vetustarum literarum sacrarumque linguarum sunt ignari, illas opiniones sequuntur, quae in publicum usum quovis modo irresperunt, quos cum alii innumeri eiusdem farinae homines sequuti sunt et quotidie misere sequuntur. [...] Ad quod tibi facile respondeo permultum interesse inter ecclesiae autoritatem aliquid certa definitione determinantem, multumque interest inter veteres historiarum sanctos scriptores aliquid suorum vel vicinorum temporum narrantes, et inter recentiores scholasticos doctores aliquid suo cerebro opinantes, fridigissimisque coniecturis contendentes. [...] o quanta canuntur et leguntur in ecclesiae officiolis, quae omnino sunt varia, ficta et falsa, ut locustas ex ovibus, saxeum pastorem, ovem in praedonis ventre balantem, redivivum ex mortuis asinum, et alia innumera, ut vix sit ulla martyris aut virginis legenda, cui non pie aliquid admentitum sit, vel vafris pessimisque nebulonibus, vel a quaestuariis fraterculis, quibus studium est, ex incauta simplicium affectionem lucrum capere, quotiens ab huiusmodi, aut anus quaedam delyra lachrimatur, aut alius quispiam pavidus inhorresca. At nunquid haec omnia approbavit ecclesia? Non certe. Quamborem quas vetustissimi sacri scriptores, divinitus corroborati, nobis commendant historias, eis indubitata fides habenda est. Iuniorum vero contraria commenta, non nisi caute et cum iudicio ecclesiae recipienda sunt ».

50 Ibid., fol. D4v-D5r: « Nihil enim de antiquitatum historiis probant recentiores et ab illarum temporibus remotissimi doctores. Itaque facile contemno ac refello quos tu tam magnis mitris producis, Hugonem cardinalem, Anthonium archiepiscopum, Petrum de Natalibus episcopum, insuper et Petrum Comestorem et doctorem Angelicum, quibus, scio, et alios innumeros addere posses, si omnes neotericorum contionatorculorum sordidos commentarios, sermones, summulas, collectanea, reportata et dictionarios recensueris. Mirorque non modicum quod vastum illum coacervatorem Vincentium cum suo speculo et illum chronicarum supplementatorem, illosque vocabulorum latrunculos Mammatrectum, Graecistam, Catholiconem in hunc exercitum tuum non vocaveris »; cf. Ibid., fol. D7r: « Ex horum numero sunt plerique iuniores, historiarum et linguarum ignari, quorum commentaria super Bibliam cum legimus, ipsa Sacra Scriptura illorum futilibus questiunculis ac impiis litigiis, tam dubia et incerta redditur, ut illorum commentis etiam ipsa [...] Christi genealogia irrisioni pateat Iudaeis. Cuiusmodi insolentes sophistae, cum ipsi regiones et loca, quae divinae literae commemorant ex cosmographia non didicerunt, gentium ritum, origines, instituta et cultum ex historiarum scriptoribus non cognoscunt, rerum autem naturalium, quarum maxima copia in Prophetis atque hinc inde in Sacris Literis mentio est et ex quarum proprietate saepe mysterii intellectus dependet, cognitione penitus careant, sola dialectica freti se satis eruditos putant, si de quavis re syllogismum in Barbara aut Baroco connectere sciant ». 
limiti della natura umana, che incontra l'esegeta nel processo di decodificazione del simbolo e illustrazione del senso figurato. A parere di Agrippa l'interprete meticoloso eviterà accuratamente di costringere la Parola di Dio al significato apparente, ossia all'involucro del messaggio rivelato, poiché altrimenti numerosi episodi della narrazione biblica risulterebbero nient'altro che storielle inconsistenti, contraddittore, persino favolose, per non dire ridicole. ${ }^{51}$ Le oscurità evidenti che spesso incontriamo nella lettura della Bibbia saranno affrontate ricorrendo alla collazione testuale, ossia chiarendo un passo oscuro del testo per mezzo di un passo analogo, affine o contestuale, che sia più perspicuo e intelligibile, sulla base al presupposto erasmiano per cui la Scrittura illustra, spiega e chiarifica sé stessa, allorché ci affidiamo al significato mistico del suo contenuto: « optima ratio est interpretandi divinas litteras, si locum obscurum ex aliorum locorum collatione reddamus illustrem et mysticam scripturam mystica item scriptura exponat $»^{52}$

Qualora la comprensione risulti pur sempre ostacolata e l'ambiguità testuale comunque invalicabile, l'esegeta ricorrerà alla tradizione, specialmente ai teologi più antichi e autorevoli che di quelle oscurità hanno saputo rintracciare un senso plausibile, in linea con un'interpretazione spirituale..$^{53}$ Nella summenzionata

51 AGRIPPA, De triplici ratione, p. 130-132: «Quod si solum literalem sensum legis apprehendas absque spiritu futurae lucis, veritatis et perfectionis, nihil erit lege magis ridiculum et anili fabulae milesiique sermonis magis simillimum ».

52 ERASMUS ROTERODAmUS, Ratio seu Methodus compendio perveniendi ad veram Theologiam, in ID., Ausgewählte Schriften, vol. III, ed. GERHARD B. WINKLER, Wissenschaftliche Buchgesellschaft, Darmstadt 1967, p. 454; cf. anche cf. ibid., p. 178: « Denique series ipsa narrationis altius repetita, sententiam aperit alias obscura. Saepenumero locorum collatio nodum explicat difficultatis, dum quod alibi dicutm est rectius, alibi dilucidius refertur "; sull'importanza della collatio nel metodo esegetico di Erasmo, cf. Nikolaus STAUBACH, "Memores pristinae perfectionis. The importance of the Church Fathers for Devotio moderna ", in IRENA BACKus (ed.), The Reception of the Church Fathers in the West. From the Carolingians to the Maurists, vol. I, Brill, Leiden - New York Köln 1997, p. 441-460. Per Agrippa cf. VAN DER PoEL, Cornelius Agrippa, p. 92: « Agrippa had a particular interest on the one hand in those authors (mainly Church Fathers) who have enquired into the profound meaning of the Scripture or who have explained the basic principles of Christian belief on the basis of the Bible, and on the other hand in authors and writings that have a mystical inclination ».

53 Si tratta, anche in questo caso, di un presupposto metodologico che si ispira ad Erasmo: cf. ERASMUS, Enchiridion militis Christiani, in ID., Ausgewählte Schriften, vol. I, ed. WernER WeLzIG, Wissenschaftliche Buchgesellschaft, Darmstadt 1968, p. 88: « Ex interpretibus divinae scripturae eos potissimum delige, qui a littera quammaxime recedunt. Cuiusmodi sunt in primis post Paulum Origenes, Ambrosius, Hieronymus, Augustinus. Video enim neotericos theologos litterae nimium libenter inhaerere et captiosis quibusdam argutiis magis quam eruendus mysteriis operam dare, quasi vero non vere dixerit Paulus legem nostram spiritualem esse »; cf. VAN DER POEL, Cornelius Agrippa, p. 264: «The true meaning of the Bible should be looked for by means of careful study of the text itself, and by means of careful evaluation of the exegetical work of those theologians who have studied the Bible in good faith and in accordance with Agrippa's epistemological principles. For Agrippa, this includes, besides the writings of a large 
Defensio, contestando sul piano metodologico la prassi esegetica e gli strumenti didattici dell'inquisitore domenicano, Agrippa affermerà:

Impara a comprendere i significati nascosti della Scrittura non dalle summulae, dalle cantiones, dalle postillae, dai dizionari e dalle orribili raccolte di questo genere in uso nelle scuole, o da alcuni autori di poco valore del passato, bensì dalla Scrittura stessa, che spiega se stessa ogni volta che il raffronto con altri passi chiarifica un luogo oscuro, nonché dai commentari degli antichi scrittori e dagli antichissimi interpreti che più da vicino si abbeverarono alle fonti. Dopo esserti allontanato dalle tue pozze fangose, devi abbeverarti alla loro purissima acqua per imparare a discernere il significato delle Scritture, a meno che il tuo palato e la tua lingua non siano a tal punto corrotti dalla bile, che l'educazione letteraria non abbia per te il suo vero sapore, bensì quello che un organo infetto porta con sé. ${ }^{54}$

Il programma ermeneutico di Agrippa richiama l'inviolabilità della Parola, che il vero teologo reputerà teoricamente inattaccabile, ossia non manipolabile secondo le pratiche esiziali, fomite di eresia e discordia nella Chiesa, promosse dai dottori scolastici. In altri termini, se da un lato si richiede al teologo di accostarsi alla religione con una mente libera da opinioni errate, dall'altro non occorre affatto che l'interprete aggiunga alla Scrittura alcuna dottrina che la Scrittura non proclami effettivamente. Egli non dimenticherà che i misteri divini sono stati insegnati solo da Cristo, non certo anche da Aristotele, e che sono stati rivelati per il tramite dello Spirito divino, non dedotti dalla sola ragione: « Ego illud intendo - scriverà ancora Agrippa nella sua Apologia - ad divinorum cognitionem non esse opus argutiis philosophorum, neque syllogismis dialecticorum, sed oportet illam haurire ex Scriptura divina, quae est regula infallibilis, semper permanes in suo vigore nativo $» .55$

L'inviolabilità delle Scritture evidenzia certamente l'autosufficienza del messaggio evangelico contro ogni contaminazione dall'esterno, ma non sottrae comunque il contenuto religioso al vaglio della critica storico-filologica, ossia al contributo della comprensione razionale. Ciò significa che l'uomo, a cui è

number of canonical writers (that is, the Church Fathers and certain medieval theologians), those writers belonging to the prisca theologia and the Hermetic tradition. Agrippa follows this method of research and observes these doctrinal restrictions in all his theological writings ».

54 AGRIPPA, Defensio propositionum, fol. E6r-v: « Disce Scripturae absconditos sensus intelligere non e scholasticis summulis, cantionibus, postillis, dictionariis et ex huius generis luridis collectaneis, aut e sordidis quibusdam et pridie natis auctoribus, sed ex seipsa semetipsam declarante, ubi locum obscurum aliorum collatio elucidat, tum ex veterum commentariis et priscis illis interpretibus qui proxime biberunt de fontibus. Horum purissima aqua, tuis faeculentis lacunis obmissis, tibi bibenda est, ut scias discernere sensus Scripturarum, nisi palatum et linguam tanta bili habeas infecta, ut bonae litterae tibi non sapiant id quod sunt, sed quod vitiatum organum secum circumfert »; cf. VAN DER POEL, Cornelius Agrippa, p. 86-87

55 ID., Apologia adversus calumnias, cap. 33, fol. H3v-H4r. 
demandato il compito di tradurre in scelta esistenziale e azione concreta il messaggio di Dio, non si limita ad accogliere pedissequamente il dettato scritturistico, così come non è legittimato a sottometterne il senso alle prescrizioni della logica aristotelica. Al contrario, l'eccellenza della Rivelazione rende al contempo la Parola di Dio inesauribile, vale a dire bisognosa di un assiduo cimento interpretativo da parte dell'uomo. L'inesauribilità del messaggio, che invoca la collaborazione dell'uomo, è il presupposto fondamentale del discorso religioso, in quanto certifica l'origine divina e la validità perpetua della Rivelazione, ma si richiama parimenti alle condizioni storiche della sua trasmissione e del suo compimento nel tempo. ${ }^{56}$ Poiché inesauribile, dunque, la Parola richiede da un lato il contributo unanime delle facoltà umane - mens (piano della fede), ratio (piano della scienza), idolum (piano dell'immaginazione) ${ }^{57}$

56 VAN DER PoEl, Cornelius Agrippa, p. 83-84: «Agrippa considered Biblical exegesis necessary, because for various reasons connected with human imperfection, the Word of God is not always communicated correctly to men. In chapter 99 of De incertitudine (On prophets), Agrippa explained this thought in a particularly expressive way by observing that even divinely inspired men such as the Old Testament prophets and the New Testament apostles and evangelists were not infallible, and occasionally failed to see the truth and made involuntary mistakes. As examples of prophets who made errors, Agrippa mentions Moses, who did not keep his promise to lead the people of Israel, Jonas, who failed to anticipate God's change of plan when he proclaimed that Nineveh would be destroyed within forty days (Jonah 3, 4-10), and Peter, who was reprimanded by St. Paul. [...] In the same chapter Agrippa also points out that even the corpus of Holy Books is not without imperfection, since some books are lost, while others have not been accepted as canonical. Some examples of the first category are the Book of the Wars of the Lord, mentioned in Numbers 21, 14, the Book of Jashar, mentioned in Joshua 10,13, and the numerous apocryphal Biblical texts »; cf. AGRIPPA, De Incertitudine \& Vanitate Scientiarum, Ioannes Grapheus, Antverpiae 1530, cap. 99, fol. Rr-R2v.

57 Sulla tripartizione dell'anima cf. AGRIPPA, De occulta philosophia, III, 43, p. 538: «Anima humana constat mente, ratione, et idolo: mens illuminat rationem, ratio fluit in idolum, omnia una sunt anima. Ratio, nisi per mentem illuminetur, ab errore non est immunis; mens autem lumen rationi non praebet, nisi lucescente Deo, primo videlicet lumine. Prima enim lux in Deo est supereminens omnem intellectum, quapropter non potest lux intelligibilis vocari; sed lux illa quando infunditur menti fit intellectualis atque intelligi potest; deinde quando per mentem infunditur rationi fit rationalis ac potest non solum intelligi, sed etiam cogitari; deinde quando per rationem infunditur in idolum animae efficitur non solum cogitabilis, sed etiam imaginabilis, nedum tamen corporea; quando vero exinde migrat in aethereum idoli vehiculum efficitur primum corporea, non tamen manifeste sensibilis, donec transierit in corpus elementale sive simplex aëreumque sive compositum, in quo efficitur lux manifeste visibilis ad oculum ». Il passo si fonda su MARSILIUS FICINUS, Theologia platonica, XIII, 4, ed. RAYMOND MARCEL, Les Belles Lettres, Paris 1964, vol. II, p. 239: "Prima lux in Deo est atque ibi est talis ut superemineat intellectum, ideoque non potest lux intelligibilis appellari. Sed lux illa Dei, cum infunditur angelo, fit e vestigio lux intellectualis atque intelligi potest. Quando infunditur animae, fit rationalis ac potest non intelligi solum, sed cogitari. Inde migrat in animae idolum, ubi fit sensitiva, nondum tamen corporea. Inde in aethereum vehiculum idoli, ubi fit corporalis, nondum tamen manifeste sensibilis. Denique in corpus elementale, sive simplex aeriumque sive compositum, quod est aetherei vasculum, in quo evadit manifeste visibilis ». 
- incaricate di cooperare, assieme alla Grazia divina, alla comprensione dei misteri religiosi; dall'altro il supporto della tradizione teologica più antica, riconosciuta depositaria degli strumenti necessari all'attività ermeneutica, ossia a formare e irrobustire la competenza dell'uomo in campo spirituale. ${ }^{58}$

IV

Il ragionamento che Agrippa sviluppa nelle pagine agguerrite del De triplici ratione, richiamate in precedenza, sottolinea il sostrato ideologico che alimenta la sua riflessione teologica. Si tratta di una concezione profondamente etica della funzione che l'uomo di lettere è chiamato a occupare; una persuasione che rimarca l'adesione matura di Agrippa agli ideali umanistici, elaborati in chiave filosofico-teologica durante il periodo italiano. Formulati più sistematicamente nelle opere della maturità, come il De incertitudine et vanitate scientiarum e il De occulta philosophia, tali ideali si appellano sia al riconoscimento della dignità umana, delle sue facoltà e delle sue scelte nel percorso di conoscenza del mondo naturale e sovrannaturale, sia a una visione della sapienza intesa come riforma delle coscienze, come ricostituzione del legame originario tra l'uomo e Dio: un programma di ricerca e di azione che, lungo tutta la vicenda biografica di Agrippa, avrà per oggetto la magia e per scopo la religione.

Nel contesto del soggiorno in Italia preoccupazione specifica del filosofo di Nettesheim è quella di rivendicare per il cultore degli studia humanitatis, vale a dire per il pensatore incaricato di riorganizzare la cultura, un impegno educativo di rilievo fondamentale: la libertà di interpretare consapevolmente le fonti della vera sapienza allo scopo di promuovere l'edificazione e il progresso della comunità in cui opera. Ė un principio metodologico che Agrippa salvaguarderà con tenacia nel corso degli anni, rilevando spesso la necessità che il pensatore cristiano partecipi attivamente allo sforzo esegetico concentrato sulle Scritture. Questa operazione ha un valore marcatamente politico, in quanto assegna al pensatore laico un ruolo fondamentale nell'elaborazione del messaggio di fede, contestando chiaramente l'autorità del teologo di professione, non più depositario esclusivo dell'ortodossia. L'entità del contributo laico è tangibile

58 VAn DER Poel, Cornelius Agrippa, p. 11: «Instead of a rationalistic ('Aristotelian') theology, which aims at providing Biblical truths through deductive reasoning, Agrippa champions a theology which aims, through inductive reasoning at securing the true meaning of the Word of God. The method of reasoning which Agrippa applied in all his writings in order to practice this theology combines [...] the comparison of similar Biblical texts (mostly on the level of their allegorical meaning, although the moral sense also enters into the range of Agrippa's interests) and the confirmation of the correct meaning by authors considered to be worthy of authority and approved by the Church ». 
soprattutto nel caso di questioni teologiche sulle quali la Chiesa non ha ancora stabilito un fondamento dogmatico:

Nella Chiesa sono state ammesse e studiate secondo un'antichissima consuetudine svariate forme di esposizione delle Sacre Scritture, senza che per questo nessuno, professandole, possa distorcere l'esposizione delle Sacre Scritture in favore di ciò che è contrario alla fede e alla Chiesa. A ciascuno è permesso approfondire le sue convinzioni, particolarmente con più libertà nelle questioni che non sono state determinate dalla Chiesa, o in relazione alle quali non è importante per la fede sostenere che siano o non siano così, o che siano altrimenti. ${ }^{59}$

Come già evidenziato, l'attività di pensiero che fonda il discorso teologico è per Agrippa decrittazione del messaggio divino, esame e scioglimento, tramite il contributo concorde di ragione, fede e immaginazione, degli enigmi che Dio ha rivelato ed esposto nel tessuto letterario delle Sacre Scritture. Lo studio biblico, a cui si sommano l'esercizio della giustizia e l'integrità della devozione, rappresenta un sentiero continuo di maturazione e perfezionamento per il cristiano, quand'anche l'esercizio interpretativo comporti l'esperienza di orizzonti semantici del tutto inconsueti. ${ }^{60}$ In casi specifici di formulazione esegetica, Agrippa non esita affatto a rivendicare la paternità di letture non tradizionali, non conformi all'ortodossia cattolica.

Si pensi, ad esempio, alla tesi rischiosa e controversa che sorregge l'opuscolo De originali peccato, risalente quasi certamente al 1518, ma pubblicato anch'esso nel 1529. L'opinione 'disputabilis dumtaxat, et fortasse non falsa' è proposta e

59 AGRIPPA, Defensio propositionum, fol. I7v: « Multiplices enim sensus exponendi Sacras Scripturas in Ecclesia recepti sunt et vetustissima consuetudine frequentati, in quibus modo nemo retorqueat Sacrae Scripturae expositionem ad id quod contrariatur fidei et Ecclesiae. Unicuique in suo sensu abundare licet, magis autem libere in his quae nondum sunt per Ecclesiam determinata, aut quorum nihil interest ad fidem utrum sint, vel non sint sic, vel aliter teneantur "; cf. ZAMBELLI, «Cornelio Agrippa, Erasmo », p. 32-33: « La polemica sulla legittimità dell'intervento dei laici e degli umanisti nelle questioni teologiche, da questi scritti del periodo centrale della vita di Agrippa continuerà e sarà sempre più fondamentale, e sempre più erasmiana, nelle opere coclusive. Essa rappresenta il filo rosso che congiunge le meditazioni occasionali sulla dignità delle donne, sul matrimonio e su certi punti dell'interpretazione evangelica con le opere conclusive, il De vanitate e le relative autodifese (Querela, Apologia, Dialogus de vanitate) che costituiscono un tragico e fermo bilancio delle sue convinzioni di fronte alle condanne e persecuzioni dei 'Theologastri' ».

60 VAN DER POEL, Cornelius Agrippa, p. 264: «More specifically, he embraces a theology which confines itself to the study of those elements in Christian revelation which remain uncertain. This implies that theologians should not debate the articles of faith and the other points concerning which there exists a dogma or binding council pronouncements embodying religious truth. In their research, theologians should aim at reaching an understanding of the profound truth concerning the uncertainties in the revelation, that is, at reaching a proper understanding of God's intentions ». 
argomentata senza riserve retoriche o cautele nicodemitiche. L'episodio descritto nel racconto di Genesi è decodificabile a tutti gli effetti in chiave allegorica: la trasgressione adamitica allude al rapporto sessuale tra l'uomo e la donna. ${ }^{61}$ Analogamente, i protagonisti del racconto simboleggiano le facoltà dell'anima e il loro rapporto in un contesto strettamente filosofico: Adamo è la fede, Eva la ragione, il Serpente i sensi e la sensualità; il peccato consiste nella volontà di offuscare la fede che, per il tramite della ragione, abbandona la sapienza e l'obbedienza a Dio al fine di assecondare la percezione sensibile, giungendo così a erigere al rango di scienza l'esperienza confusa dei sensi. ${ }^{62}$ Nella lettera dedicatoria che accompagna il testo dell'orazione, Agrippa chiarisce il suo punto di vista, rivendicando la libertà di dibattere su quei luoghi della Scrittura relativamente ai quali né la dottrina della Chiesa né la tradizione patristica hanno espresso un giudizio unanime e concorde, cristallizzando così un profilo dogmatico:

Varie furono le teorie sul peccato originale e sulla prima trasgressione dell'uomo fra gli interpreti più dotti e insigni delle Sacre Scritture, sia fra gli antichi che fra $\mathrm{i}$ più recenti. Avendo spesso riflettuto su queste teorie, mi sembrava che essi non avessero scritto altro che cose oscure e ambigue. Pertanto, dopo aver tralasciato, non certo trascurato, le loro opinioni, presenterò una mia opinione, nuova $\mathrm{e}$ diversa, perlomeno discutibile e forse non falsa. Non so se qualcuno prima di me abbia formulato proprio questa opinione. Qualora qualcuno l'abbia fatto, non lo offenderò certo se ne rivendicherò la paternità, nella misura in cui con l'impegno del mio ingegno e tramite lo studio delle Scritture ho sottratto questa opinione alle tenebre della negligenza. ${ }^{63}$

61 AgRIPPA, De originali peccato, in ID., De Nobilitate \& Praecellentia Foeminei sexus, ad Margaretam Augustam Austriaco(rum) et Burgundionum Principem..., Michael Hillenius in Rapo, Antverpiae 1529, fol. I5v: « Ipsa autem opinio nostra talis est, non aliud fuisse originale peccatum, quam carnalem copulam viri et mulieris ».

62 Ibid., fol. I4r-v: "Erant in Paradiso Adam et Eva, erat in eo etiam serpens callidior cunctis animantibus terrae. Adam quidem fides est stabilita in Deo, fundamentum rationis. Ideo ex costa Adae creata est Eva, ratio libera. Serpens autem est ipsa sensualitas quae serpit in terrenis, caducis, infimis et carnalibus. Hic itaque serpens per concupiscentiam sensibilium tentavit Evam, rationem scilicet, quae acquiescens serpenti, ipsis puta sensuum illecebris, decepta est; quae deinde per discursum argumentativum in sensibilibus decepit virum Adam, deiciens fidem, quae delapsa in sensibilia, nihil plus veri credens, quam ex sensibilibus per rationem demonstrari potest. Defecit adeo divinaque cognitione et contemplatione, ad quam creata est a Deo ». Per quanto riguarda l'ipotesi esegetica che sorregge l'opuscolo di Agrippa rinvio a VAN DER POEL, «Agrippa von Nettesheim and Rhetoric », p. 177-206; cf. anche ID., Cornelius Agrippa, p. 233-245.

63 AGRIPPA, De originali peccato, fol. I3r: «De originali peccato primaque hominis transgressione apud doctissimos praestantissimosque tam veteres quam recentiores Sacrarum Litterarum interpretes variae feruntur sententiae, quas animo mecum cum saepe revolverem, nihil mihi praeter obscura quaedam et ambigua scripsisse videbantur. Non contemptis igitur sed relictis eorum opinionibus, aliam novamque et meam opinionem (disputabilem dumtaxat, et fortasse 
Il valore antidogmatico di questo approccio è rimarcato nella volontà di non asserire, cioè di non presentare articoli di fede o di scienza, bensì di avanzare opinioni, ${ }^{64}$ ossia discutere argomenti passibili di critica, confutazione o rettifica, comunque maturati tramite il libero esercizio facoltà razionale nell'analisi delle Scritture. ${ }^{65}$

Molti teologi, sia antichi sia più recenti, hanno elaborato numerose opinioni su questo argomento [scil. il peccato originale] e ciascuno di essi approfondisce le sue convinzioni. Non so se sia meritevole di indulgenza il fatto di aggiungere alle loro la mia opinione; opinione dico, non fede, non scienza. Se essa risulterà errata, non sarò quindi considerato uno che erra nella vera fede e che viola la pura sapienza cristiana. Stando così le cose, mi è lecito esprimere liberamente un'opinione. $\mathrm{E}$ qualora io - poiché sono un uomo di età immatura e dotato di poca intelligenza e istruzione - non riesca a fornire un numero sufficiente di testimonianze scritturali, così come richiede la questione, per illustrare la mia interpretazione, forse mi seguiranno alcuni uomini più dotti di me, i quali approveranno la mia opinione. Confido, infatti, sul fatto che mi sosterranno non poco nella misura in cui confermeranno la mia opinione con argomenti e ragioni più valide. ${ }^{66}$

non falsam) adferam. Nescius si quispiam ante me eandem opinatus sit, qui si quis est, nullam facio illi iniuriam si hanc opinionem meam esse dixero, quatenus illam non aliunde quam proprii ingenii diligentia Scripturarumque examine ex negligentiae tenebris eripuerim ».

64 VAN DER POEL, Cornelius Agrippa, p. 179-180: «In other words, the term 'opinio' denotes exactly the dialectical nature of Agrippa's theological writings. In fact, Agrippa had used this term in De originali peccato to explain that this declamation was meant as a contribution to the debate on Original Sin. [...] In De originali peccato, Agrippa also uses the term 'opinio' to express the intellectual modesty with which he formulates his contribution to the debate. More specifically, he uses it to clarify the dialectical nature of his treatise and to declare his willingness to submit it to the authority of the Church ».

65 ZAmbelLI, «Humanae litterae, verbum divinum, docta ignorantia negli ultimi scritti di Enrico Cornelio Agrippa », Giornale critico della filosofia italiana, 45 (1966), p. 209: « La lezione erasmiana aveva insegnato ad Agrippa che anche i testi a cui entrambi riconoscevano autorità e dignità sovrumane erano frutto della stesura di uomini che ne erano stati strumenti imperfetti, e fallibili secondo la natura umana; il risultato della loro elaborazione era dunque soggetto, esso pure, alle leggi della critica storica: [...] Le contraddizioni e la fallibilità dei testi sacri, sottolineando la differenza essenziale fra il divino e l'umano, sono ricondotte esse stesse a scopi apologetici; e questa impostazione presenta la critica storica, ispirata a Valla e ad Erasmo, su un fondamento assai opportuno per quei tempi. Gerolamo medesimo riconosceva che gli Apostoli e gli Evangelisti avevano talvolta commesso errori di memoria. Parimenti Agostino pensava che gli Apostoli ignorassero qualche fatto e s'ingannassero in qualche caso, e che persino Pietro avesse errato in materia di fede e fosse stato corretto da Paolo ».

66 AGRIPPA, De originali peccato, fol. I5v: « Multi et veteres et recentiores theologi super hac re multa opinati sunt et unusquisque eorum in suo sensu abundat, quiscum nescio an plane venia dignum sit meam quoque opinionem conferre, opinionem dico, non fidem, non scientiam, ut si erronea fuerit opinio mea, ab errore verae fidei ac incontaminatae christianae sapientiae praevaricatione sim alienus. Atque hac conditione nunc libere opinari licebit et si (ut enim homo sum immaturae aetatis, ingenii ac litteraturae minoris) in sensu meo non tantis 
Sul piano educativo il metodo che Agrippa elabora per rinnovare la riflessione teologica prevede in primo luogo una formazione adeguata, ossia l'acquisizione della competenza dottrinale, letteraria, linguistica, storica necessaria per accedere alla testualità della Parola di Dio; in secondo luogo l'insegnamento, vale a dire l'esercizio di esposizione e commento delle Scritture, che si realizza nella discussione aperta, non dogmatica, non ignara delle fonti e sprezzante della tradizione, condotta criticamente sul testo sacro; in terzo luogo, la conversione spirituale, un passaggio peculiarmente filosofico. 'Conversione' significa in Agrippa un atto di coscienza grazie al quale l'anima dell'uomo giunge a purificarsi dall'ignoranza, dall'errore e dal peccato, scegliendo di amare Dio e la sua sapienza. ${ }^{67}$ La conversione certifica, dunque, in un'esperienza tangibile e condivisibile il fine ultimo della teologia, il processo di rigenerazione spirituale dell'uomo.

Il programma di Agrippa assume così i caratteri di una riforma condivisa, programmaticamente rivolta all'intera comunità e incentrata, per l'appunto, sulla discussione e trasmissione dei testi che custodiscono una sapienza salvifica, capace di risvegliare le coscienze e di tradursi in una prassi di rinnovamento del vincolo sociale. ${ }^{68}$ Il percorso sapienziale, cioè lo studio e l'apprendimento delle

Scripturarum testimoniis abundem, ut res ipsa requirit, forsitan sequentur me doctiores quidam, quibus haec nostra opinio non displicebit: ab his me non modicum adiuvari posse confido quatenus validioribus rationibus ac argumentis suis opinionem nostram robustiorem effecerint ».

67 Cf. ID., De triplici ratione, p. 142: « Anima itaque nostra, carne inclusa corruptibili nimioque eius demersa commercio [FICINUS, Theol. plat. IX, 2, ed. MARCEL, vol. II, p. 11], nisi viam carnis superaverit fueritque pristinam naturam sortita evaseritque mens pura, quasi par angelo, frustra laborat in divinis. Sed quae anima haec est, nisi quae indubia spe et superni numinis desiderio phantasiam silere iubet? Et quae, veram fidem firmiter amplexa, assuetis rationis naturalis discursibus ammodo non confidit, et quae, ardenti amore adhaerens Deo, sola vivit mente, evasit angelus, capit toto pectore Deum? [FICINUs, Theol. plat. XIII, 4, ed. MARCEL, vol. II, p. 237 ]».

68 Ciò vale soprattutto per la magia: cf. CHARLES ZIKA, Exorcising our Demons. Magic, Witchcraft and Visual Culture in Early Modern Europe, Brill, Leiden 2003 (Studies in Medieval and Reformation Thought, 91), p. 150-151: « The most critical and distinctive characteristic of Agrippa's occult philosophy or magic, [...] was its concern with 'operation'. Magic did not involve a mystical escape from society and the world for scholars such as Agrippa - certainly not from the courtly and academic world which could provide them with status and prestige, and in Agrippa's case, also not from the world of the practicing physician, orator and advocate. The ultimately successful denigration of 'magic' in later decades and centuries, in ways which Agrippa was endeavouring to resist, makes it difficult for us to accept this social element in sixteenthcentury understanding of the term. Macig involved a knowledge which could 'operate' in the world, which could influence individual consciousness, provide models for group behavior, help ensure future success, mitigate the influence of evil and misfortune. And in this sense it shared common aims with the humanists, who promoted the rhetorical and literary skills of anquity and were concerned to influence behavior through eloquence and persuasions. Both 
verità che accompagnano l'anima del cristiano alla salvezza, risulta pienamente accessibile a chiunque decida di intraprenderlo: «Omnis itaque homo - ribadisce Agrippa - potest Deum cognoscere, si velit.$^{69}$ A ben vedere, tale puntualizzazione rimarca con efficacia, al di là delle divergenze di approccio sottolineate in precedenza, l'affinità intellettuale che lega indissolubilmente la riforma di Agrippa alla lezione di Erasmo. Come insegnava lo stesso Erasmo, organizzando l'impianto teorico della philosophia Christi che avrebbe fondato il Novum Instrumentum, la celeberrima edizione aggiornata del Nuovo Testamento (1516), il campo della teologia non è privilegio dottrinale riservato ai dotti o ai teologi di professione, bensì assume un valore fondamentale per l'educazione di tutta la società cristiana, giacché il verbo di Dio è patrimonio aperto a tutti gli uomini, di qualunque condizione essi siano: 'L'essere dotti - scrive Erasmo - è riservato a pochi, mentre a nessuno è negato di essere cristiano, di avere fede e oserei perfino dire che a nessuno è negato di essere teologo'. ${ }^{70}$ Questa lezione, che Agrippa trae in modo particolare dallo studio della Paraclesis ad lectorem pium di Erasmo, resterà viva e operante anche molti anni più tardi, nelle pagine radicali del De vanitate in cui l'autore vorrà definire la missione educativa del teologo:

Non crediate che la parola di Dio spetti solo ai teologi, ma ad ogni essere umano, uomo, donna, vecchio, giovane, bambino, indigeno, straniero o forestiero. [...] Tutta la teologia deve essere comune a tutti i fedeli, ma a ciascuno secondo la capacità e la misura del dono dello Spirito santo. È dunque compito del buon dottore distribuire a ciascuno in base a quanto ognuno è capace di comprendere, all'uno in latte, all'altro in solido cibo, e non privare nessuno del pasto della verità necessaria. $^{71}$

In conclusione, considerando la vicenda intellettuale di Agrippa, il soggiorno di formazione in Italia costituisce una stagione altamente significativa, un'occasione

conceptions of knowledge privileged the uses to which knowledge was put over the coherence of the system throught which it was enunciated ».

69 AGRIPPA, De triplici ratione, p. 112.

70 Erasmus, Paraclesis, LB, V, col. 141, in Fiorella De Michelis PinTacuda, Tra Erasmo e Lutero, Edizioni di Storia e Letteratura, Roma 2001 (Studi e testi del Rinascimento europeo, 11), p. 163.

71 AgripPa, De Vanitate , cap. 100, fol. S2r-v: « Non est ergo, ut putetis, ipsum [scil. verbum Dei] ad solos Theologos pertinere, sed ad omnem hominem, sive vir, sive mulier, sive senex, sive iuvenis, sive puer, sive indigena, sive advena, sive proselytus [...]. Quin tota ipsa Theologia omnibus fidelibus communis debet, unicuique autem secundum capacitatem et mensuram donationis Spiritus sancti. Boni itaque doctoris est unicuique secundum id quod confert quantum capere potest distribuere, illi quidem in lacte, alteri autem in solido cibo [Hebr., 5, 12], et neminem necessarie veritatis pastu defraudare »; cf. PERRONE COMPAGNI, «L'innocenza di Eva. Retorica e teologia nel De nobilitate foeminei sexus di Agrippa », Bruniana \& Campanelliana, 12/1 (2006), p 79: « La teologia non è ambito riservato a professionisti, ma è cosa di tutti - perché è sapere isagogico, che inquadra la vita umana in una prospettiva ultramondana e nello stesso tempo dirige la ragione mondana e la rende legittima e fruttuosa ». 
per elaborare premesse, definire obiettivi, impostare un programma culturale che segnerà coerentemente anche gli sviluppi successivi. Nel corso di un periodo così ricco di meditazione ed elaborazione teorica - caratterizzato, tra l'altro, da numerose letture ermetiche, neoplatoniche, cabalistiche, lulliane - emerge marcatamente il profilo ideologico di Agrippa, oggi alquanto distante dal ritratto contraddittorio che la storiografia novecentesca ha delineato in alcuni casi: non più un occultista enciclopedico, compilatore di testi magici, preoccupato soltanto divulgare le discipline esoteriche in un sistema compiuto, ${ }^{72}$ non più un critico mordace delle scienze e delle arti, rivolto a screditare beffardamente il sapere umano di fronte alla superiorità incontrovertibile delle Sacre Scritture $;^{73}$ bensì un appassionato fautore delle humanae litterae ${ }^{74}$ teologo e riformatore religioso ${ }^{75}$ impegnato nello studio sistematico degli antichi filosofi e nel recupero della riflessione religiosa che l'antichità ha trasmesso; insomma un pensatore del Rinascimento, interessato a riorganizzare il sapere scientifico attraverso una proposta filosofica che, rifacendosi alla religiosità e all'antropologia di carattere ermetico-neoplatonico, sappia congiungere magia e teologia in un tragitto sapienziale comune, destinato a concludersi nella palingenesi della comunità dei Cristiani.

72 Lynn ThorndiKe, A History of Magic and Experimental Science, vol. VI, Columbia University Press, New York 1923-1941, p. 129-131; FRANCIS A. YATES, Giordano Bruno and the Hermetic Tradition, Routledge and Kegan Paul, London 1964, p. 130.

73 Richard H. Popkin, The History of Scepticism from Erasmus to Descartes, Koninklijke Van Gorcum \& Comp. N. V., Assen 1960, p. 24-25; NAUERT, Agrippa and the Crisis, p. 304.

74 ZAMBELLI, « Cornelio Agrippa nelle fonti », p. 173.

75 Rosamaria AlibRANDI, «Magia, filosofia occulta e Inquisizione. Il lato oscuro della modernità», Heliopolis, 10/1 (2012), p. 9: «Il vero problema di Agrippa, che vive intensamente gli anni della Riforma, sembra dunque essere quello religioso [...]. La riforma della magia per Agrippa deve condurre alla restaurazione di una ars miraculorum che la renda operativa in tutti i campi del sapere recuperando la sua matrice originariamente religiosa, poiché la scienza e la ragione si sono allontanate dalla fonte originaria della sapienza ». 
Dario Gurashi

Bibliografia

Agrippa, Heinrich Cornelius, Dehortatio gentilis theologiae, in Henrici Cornelii Agrippae De Nobilitate \& Praecellentia Foeminei sexus, ad Margaretam Augustam Austriaco(rum) et Burgundionum Principem..., Michael Hillenius in Rapo, Antverpiae 1529.

- De originali peccato, in Henrici Cornelii Agrippae De Nobilitate \& Praecellentia Foeminei sexus, ad Margaretam Augustam Austriaco(rum) et Burgundionum Principem..., Michael Hillenius in Rapo, Antverpiae 1529.

- De Incertitudine \& Vanitate Scientiarum \& Artium atque excellentia Verbi Dei Declamatio, Ioan[nes] Grapheus, Antverpiae 1530.

- Apologia aduersus calumnias propter Declamationem de Vanitate scientiarum, \& excellentia verbi Dei, sibi per aliquos Louanienses Theologistas intentatas, in Apologia aduersus calumnias... Querela super calumnia, ob eandem Declamationem, sibi per aliquot scleratissimos sycophantas, apud Caesaream Maiest[atem]. Nefarie ac proditorie illata, 1533, fol. A7r-Kv.

- Defensio propositionum praenarratarum contra quendam Dominicastrum earundem impugnatorem..., in Id., De beatissimae Annae monogamia, ac unico puerperio propositiones abbreuiatae \& articulatae... Eiusdem Agrippae defensio propositionum praenarratarum contra quendam Dominicastrum earundem impugnatorem.... Quaedam epistolae super eadem materia..., 1534, fol. B4r-M8v.

- Oratio in Praelectionem Convivij Platonis, in Orationes X... Eiusdem de duplici coronatione Caroli V. Caesaris apud Bononiam, historiola. Eiusdem, ac aliorum doctorum virorum epigrammata, Ioannes Soter, Coloniae 1535, fol. A2r-B3r.

-- Oratio habita Papiae in praelectione Hermetis Trismegisti, de potestate \& sapientia dei. ANNO M. D. XV, in Paola Zambelli, "Cornelio Agrippa. Testi scelti», in Eugenio Garin, Mirella Brini, Cesare Vasoli, Paola Zambelli (eds.), Testi umanistici su l'ermetismo. Testi di Ludovico Lazzarelli, F. Giorgio Veneto, Cornelio Agrippa di Nettesheim, Fratelli Bocca, Roma 1955 [Archivio di filosofia: organo della Società filosofica italiana, 1/1 (1955)], p. 105-162.

- Dialogus de homine, in Paola Zambelli, "Agrippa di Nettesheim. Dialogus de homine», (prima ed.), Rivista critica di storia della filosofia, 13 (1958), p. 47-71.

- De occulta philosophia libri tres, ed. Vittoria Perrone Compagni, Brill, Leiden New York - Köln 1992 (Studies in the History of Christian thought, 48). 
- De triplici ratione cognoscendi Deum, in Vittoria Perrone Compagni, Ermetismo e Cristianesimo in Agrippa. Il 'De triplici ratione cognoscendi Deum', Polistampa, Firenze 2005 (Hermetica Mediaevalia, 3).

Alibrandi, Rosamaria, « Magia, filosofia occulta e Inquisizione. Il lato oscuro della modernità », Heliopolis, 10/1 (2012), p. 7-12.

Celenza, Cristopher S., Il Rinascimento perduto. La letteratura latina nella cultura italiana del Quattrocento, Carocci, Roma 2004 (Lingue e letterature Carocci, 176).

Champerius, Symphorianus, Commentarium in Asclepij Mercurij Trismegisti discipuli diffinitiones theologicas ad Ammonem regem, ed. Cesare Vasoli, in Enrico Castelli (ed.), Umanesimo ed Esoterismo. Atti del 5. Convegno internazionale di studi umanistici, Oberhofen, 16-17 settembre 1960, CEDAM, Padova 1960 [Archivio di filosofia: organo della Società filosofica italiana, 2/3 (1960)], p. 260-289.

De Michelis Pintacuda, Fiorella, Tra Erasmo e Lutero, Edizioni di Storia e Letteratura, Roma 2001 (Studi e testi del Rinascimento europeo, 11).

Ebgi, Raphael (ed.), Umanisti italiani. Pensiero e Destino, Einaudi, Torino 2016 (I millenni).

Ficinus, Marsilius, Argumentum Marsilij Ficini Florentini in librum Mercurij Trismegisti ad Cosmum Medicem, in Contenta in hoc volumine. Pimander Mercurii Trismegisti liber de sapientia et potestate Dei. Asclepius Eiusdem Mercurii Liber de Voluntate Diuina. Item Crater Hermetis, a Lazarelo Septempedano, Paris 1505, fol. 2r-3v.

- Apologia, in ID., Opera omnia, Henricuspetrus, Basileae 1576, t. I.

- Epistolarum libri XII, in ID., Opera omnia, Henricuspetrus, Basileae 1576, t. I.

- Theologia platonica de immortalitate animorum = Théologie platonicienne de l'immortalité des âmes, ed. Raymond Marcel, 3 vols., Les Belles Lettres, Paris 19641970.

Hanegraaff, Wouter. J., «Better than Magic. Cornelius Agrippa and Lazzarellian Hermetism », Magic, Ritual and Witchcraft, 4/1 (2009), p. 1-25.

- « Heinrich Cornelius Agrippa », in Christopher Partridge (ed.), The Occult World, Routledge, London-New York 2016, p. 91-98.

Keefer, Michael. H., «Agrippa's Dilemma: Hermetic 'Rebirth' and the Ambivalences of De vanitate and De occulta philosophia », Renaissance Quarterly, 41 (1988), p. 614-653.

Lazarelus, Lodovicus, Lodouici Lazareli Poete Christiani ad Ferdinandum Regem dialogus cui titulus Crater Hermetis, in Contenta in hoc volumine. Pimander Mercurii Trismegisti liber de sapientia et potestate Dei. Asclepius Eiusdem Mercurii Liber de 
Voluntate Diuina. Item Crater Hermetis, a Lazarelo Septempedano, Paris 1505, fol. 60v$81 \mathrm{v}$.

Lehrich, Christopher I., The Language of Demons and Angels. Cornelius Agrippa's Occult Philosophy, Leiden, Brill 2003 (Brill's Studies in Intellectual History, 119).

Leinkauf, Thomas, "Prisca scientia versus prisca sapientia. Zwei Modelle des Umgangs mit der Tradition am Beispiel des Rückgriffs auf die Vorsokratik im Kontext der frühneuzeitlichen Debatte und der Ausbildung des Kontinuitätsmodell der prisca sapientia bzw. philosophia perennis ", Mediterranea. International Journal on the Transfer of Knowledge, 2 (2017), p. 121-143.

Mercurii Trismegisti Pimander. Liber de sapientia et potestate Dei Marsilio Ficino interprete, in Contenta in hoc volumine. Pimander Mercurii Trismegisti liber de sapientia et potestate Dei. Asclepius Eiusdem Mercurii Liber de Voluntate Diuina. Item Crater Hermetis, a Lazarelo Septempedano, Paris 1505, fol. 4r-27v.

- Corpus Hermeticum. Asclepius, ed. Arthur D. Nock, André M. J. Festugière, 2 vols., Les Belles Lettres, Paris 1945.

Morley, Henry, Cornelius Agrippa. The life of Henry Cornelius Agrippa von Nettesheim, doctor and knight, commonly known as a magician, 2 vols., Chapman and Hall, London 1856.

Nauert, Charles G., " Magic and Skepticism in Agrippa's thought », Journal of the History of Ideas, 18/2, 1957, p. 161-182.

- "Agrippa in Renaissance Italy: the Esoteric Tradition», Studies in the Renaissance, 6 (1959), p. 195-222.

- Agrippa and the Crisis of Renaissance Thought, University of Illinois Press, Urbana 1965 (Illinois Studies in the Social Sciences, 55).

Perrone Compagni, Vittoria, « Una fonte di Cornelio Agrippa: il De harmonia mundi di Francesco Zorzi », Annali dell'Istituto di filosofia, 4 (1982), p. 45-74.

- «Il De Occulta Philosophia di Cornelio Agrippa », in Guido Canziani, Gianni Paganini (eds.), Le edizioni dei testi filosofici e scientifici del '500 e del '600. Problemi di metodo e prospettive di ricerca. Gargnano sul Garda, 13 aprile 1985, Franco Angeli, Milano 1986 (Filosofia e scienza nel Cinquecento e nel Seicento. Serie 1, Studi, 29), p. 99-111.

- «Introduction », in Cornelius Agrippa ab Nettesheim, De occulta philosophia libri tres, Leiden - New York - Köln, Brill 1992 (Studies in the History of Christian thought, 48), p. 1-50.

- « Riforma della magia e riforma della cultura in Agrippa », I Castelli di Yale, 2/2 (1997), p. 115-140. 
- «Dispersa intentio: Alchemy, Magic and Scepticism in Agrippa », Early Science and Medicine, 5/2 (2000), p. 160-177.

- «Astrologia e filosofia occulta in Agrippa », Rinascimento, 41 (2001), p. 93-111.

- « Abracadabra: le parole della magia (Ficino, Pico, Agrippa)», Rivista di estetica, 42 (2002), p. 105-130.

- Ermetismo e Cristianesimo in Agrippa. Il 'De triplici ratione cognoscendi Deum', Polistampa, Firenze 2005 (Hermetica Mediaevalia, 3).

- «L'innocenza di Eva. Retorica e teologia nel De nobilitate foeminei sexus di Agrippa », Bruniana \& Campanelliana, 12/1 (2006), p. 59-80;

- «Il De occulta philosophia di Cornelio Agrippa. Parole chiave: uomomicrocosmo, prisca theologia, cabala, magia », Bruniana \& Campanelliana, 13/2, 2007, p. 429-448.

- «Tutius ignorare quam scire. Cornelius Agrippa and Scepticism», in Gianni Paganini, José R. Maia Neto (eds.), Renaissance Scepticisms, Springer, Dordrecht 2009 (Archives internationales d'histoire des idées, 199), p. 91-110.

Popkin, Richard H., The history of Scepticism from Erasmus to Descartes, Koninklijke Van Gorcum \& Comp. N. V., Assen 1960.

Prost, Auguste, Les sciences et les arts occultes au xvi e siècle: Corneille Agrippa. Sa vie et ses oeuvres, 2 vols., Champion, Paris 1882.

Reuchlin, Johannes, Capnion seu De Verbo Mirifico, s. t. 1. a. [J. Amerbach, Basileae 1494].

- De verbo mirifico. Das wundertätige Wort (1494), ed. Widu-Wolfgang Ehlers et al., Fromann-Holzboog, Stuttgart - Bad Cannstatt 1996.

- De arte cabalistica libri III, apud Thomam Anshelmum, Hagenau 1517.

Ritius, Augustinus, De motu octavae sphaerae [...] Item ejusdem Epistola de astronomie auctoribus, in aedibus Joannis de Ferrariis, Tridini 1513.

Roterodamus, Erasmus, Enchiridion militis Christiani, in vol. I, ed. Werner Welzig, Wissenschaftliche Buchgesellschaft, Darmstadt 1968.

- Ratio seu Methodus compendio perveniendi ad veram Theologiam, in Id., Ausgewählte Schriften, vol. III, ed. Gerhard B. Winkler, Wissenschaftliche Buchgesellschaft, Darmstadt 1967.

Staubach, Nikolaus, «Memores pristinae perfectionis. The importance of the Church Fathers for Devotio moderna», in Irena Backus (ed.), The Reception of the Church Fathers in the West. From the Carolingians to the Maurists, vol. I, Brill, Leiden - New York - Köln 1997, p. 405-469. 
Thorndike, Lynn, A History of Magic and Experimental Science, 8 vols., Columbia University Press, New York, 1923-1941.

Van der Poel, Marc, « Agrippa von Nettesheim and Rhetoric: An Examination of the Declamatio de originali peccato », Humanistica Lovaniensia, 39 (1990), p. 177-206.

- Cornelius Agrippa. The Humanist Theologian and His Declamation, Brill, Leiden 1997 (Brill's Studies in Intellectual History, 77).

Venetus, Franciscus Georgius, De harmonia mundi totius cantica tria, Bernardinus de Vitalibus, Venetiis 1525.

Walker, Daniel P., The Ancient Theology. Studies in Christian Platonism from the Fifteenth to the Eighteenth Century, Duckworth, London 1972.

Zambelli, Paola «Cornelio Agrippa. Testi scelti », in Eugenio Garin, Mirella Brini, Cesare Vasoli, Paola Zambelli (eds.), Testi umanistici su l'ermetismo. Testi di Ludovico Lazzarelli, F. Giorgio Veneto, Cornelio Agrippa di Nettesheim, Fratelli Bocca, Roma 1955 [Archivio di filosofia: organo della Società filosofica italiana, 1/1 (1955)], p. 105-162.

- « Agrippa di Nettesheim. Dialogus de homine, prima ed. », Rivista critica di storia della filosofia, 13/1 (1958), p. 47-71.

- "A proposito del De vanitate scientiarum et artium di C. Agrippa », Rivista Critica di Storia della Filosofia, 15/2 (1960), p. 166-180.

- «Cornelio Agrippa. Scritti inediti e dispersi », Rinascimento, 5 (1965), p. 195-323.

- «Cornelio Agrippa nelle fonti e negli studi recenti », Rinascimento, 8 (1968), p. 169-199;

- «Cornelio Agrippa, Erasmo e la teologia umanistica», in Rinascimento, 10 (1970), p. 29-88;

- «Magic and Radical Reformation in Agrippa of Nettesheim », in Journal of the Warburg and Courtauld Institutes, 39 (1976), p. 69-103.

Zika, Charles, Exorcising our Demons. Magic, Witchcraft and Visual Culture in Early Modern Europe, Brill, Leiden 2003. 